Math. Nachr. 224 (2001), 75-104

\title{
Sobolev Spaces on Non-Smooth Domains and Dirichlet Forms Related to Subordinate Reflecting Diffusions
}

By WALter FARKAS of Munich and NiELS JACOB of Swansea

Dedicated to Professor HANS TRIEBEL on the occasion of his 65.th birthday

(Received January 24, 2000; revised version August 7, 2000; accepted September 29, 2000)

Abstract. Let $\Omega$ be a bounded domain with fractal boundary, for instance von Koch's snowflake domain. First we determine the range and the kernel of the trace on $\partial \Omega$ of Sobolev spaces of fractional order defined on $\Omega$. This extends some earlier results of H. WALlin and J. MARschall. Secondly we apply these results in studying Dirichlet forms related to subordinate reflecting diffusions in nonsmooth domains.

\section{Introduction}

Let $\Omega \subset \mathbb{R}^{n}$ be an open bounded set and consider the classical Dirichlet form

$$
\mathcal{E}(u, v)=\int_{\Omega} \nabla u(x) \cdot \nabla v(x) d x, \quad u, v \in H^{1}(\Omega) .
$$

Here $H^{1}(\Omega)$ is the standard real Sobolev space. In order that a stochastic process on $\bar{\Omega}$ can be associated with the Dirichlet form $\left(\mathcal{E}, H^{1}(\Omega)\right)$ a regularity assumption is necessary, see [29] or [27], i.e. one has to assume that $H^{1}(\Omega) \cap C(\bar{\Omega})$ is dense in $\left(H^{1}(\Omega),\left\|\cdot \mid H^{1}(\Omega)\right\|\right)$ as well as in $\left(C(\bar{\Omega}),\left\|\cdot \mid L_{\infty}(\Omega)\right\|\right)$. In this case the associated stochastic process is called a reflecting Brownian motion and many papers are devoted to the detailed study of a pathwise characterization of this process, see [6], [7], [14], [27], [28], [30], [31] to mention only some of them.

Some authors considered partly the more general Dirichlet form

$$
\widetilde{\mathcal{E}}(u, v)=\sum_{k, l=1}^{n} \int_{\Omega} a_{k l}(x) \frac{\partial u}{\partial x_{k}}(x) \cdot \frac{\partial v}{\partial x_{l}}(x) d x
$$

1991 Mathematics Subject Classification. 31C25, 46E35, 60J50.

Keywords and phrases. Dirichlet form, $(\varepsilon, \delta)$ domain, trace operator, Weyl decomposition, Douglas integral, subordination in the sense of Bochner, reflecting diffusion. 
with domain $H^{1}(\Omega)$ where $a_{k l}=a_{l k} \in L_{\infty}(\Omega)$ (at least) satisfy the uniform ellipticity condition

$$
\sum_{k, l=1}^{n} a_{k l}(x) \xi_{k} \xi_{l} \geq \lambda_{0}|\xi|^{2}, \quad x \in \mathbb{R}^{n} \quad \text { and } \quad \xi=\left(\xi_{1}, \ldots, \xi_{n}\right) \in \mathbb{R}^{n}, \quad \lambda_{0}>0 .
$$

The aim is to prove a Skorohod decomposition for the diffusion $\left(X_{t}\right)_{t \geq 0}$ associated to this Dirichlet form. This involves in particular the local time of $X_{t}$ on the boundary. In [27] M. Fukushima characterized these sets $\Omega$ where such a Skorohod decomposition holds for reflecting Brownian motions: they are Cacciopoli sets (or sets with finite perimeter), see Subsection 2.1 below for a brief presentation of those sets.

In the paper [36] the second named author jointly with R. L. Schilling examined the question how to characterize subordinate reflected elliptic diffusions. More precisely, they investigated the processes corresponding to fractional powers (of order $\alpha, 0<\alpha<1$ ) of the generator of the form $\widetilde{\mathcal{E}}$. They assumed the boundary $\partial \Omega$ of $\Omega$ to be smooth. Using techniques from potential theory as well as some complex interpolation results they could first of all obtain an orthogonal decomposition of the subordinate Dirichlet forms and a corresponding boundary Dirichlet form. The construction however gives only a non-trivial result for $\frac{1}{2}<\alpha<1$. In this case it is also possible to describe the subordinate reflected diffusion as well as the boundary process as processes associated with the corresponding Dirichlet forms. The Skorohod decomposition was obtained just by subordinating the Skorohod decomposition of the original reflecting diffusion.

As already mentioned, if the Dirichlet space under consideration is not regular, no process can in general be associated with the Dirichlet form. In this case it is however possible to consider the Dirichlet form $\widetilde{\mathcal{E}}$ on the closure in $H^{1}(\Omega)$ of the restrictions $u \mid \bar{\Omega}$ of elements in $C_{0}^{\infty}\left(\mathbb{R}^{n}\right)$ to get a process.

The aim of this paper is twofold.

First we want to give a detailed introduction (not only for probabilists) to Sobolev spaces over non-smooth domains. This is done in Section 2 and in Section 3. Note that some of our results such as the trace theorems (see Theorem 3.5 and Theorem 3.9) seem to be new even for function spaces theorists. These theorems are related to the results of L. I. HEDBerg and Yu. V. Netrusov on the "spectral synthesis theorem" as presented in the book [1].

Secondly we will use these results to study those Dirichlet forms obtained by taking fractional powers of $\left(\widetilde{\mathcal{E}}, H^{1}(\Omega)\right.$ ) (now with smooth coefficients $a_{k l}$ ). In particular we want to study its orthogonal decomposition and we want to construct the boundary Dirichlet form. This is however not always possible.

The problem is that the domain $\Omega$ under consideration must have a certain minimal smoothness property and its boundary must be a $d$-set, $n-1 \leq d<n$, see Section 2 for the definition. This assumption guarantees already an orthogonal decomposition but the range of $\alpha$ is now depending on $d$, i. e. we have a non-trivial decomposition only for $\frac{n-d}{2}<\alpha \leq 1$, see Theorem 4.6. Moreover, these assumptions are sufficient to be able to associate a boundary Dirichlet form with the subordinate Dirichlet form, see Theorem 4.9. This result reflects of course in case of the subordinate situation the problem discussed before for the diffusion case. 
It should be noted that the probabilistic considerations made in Sections 6 and 7 of [36] do depend mainly on two facts: the properties of the local time of the diffusion on the boundary and the capacity of the boundary with respect to the symmetric stable processes. So far it is not clear which assumptions on the smoothness of $\partial \Omega$ and the range of $\alpha \in(0,1)$ are necessary to assume that the boundary measure is a smooth measure for the symmetric stable process of order $\alpha$, and that we may construct a positive continuous additive functional which has the boundary measure as a Revuz measure.

The notation is standard. Following H. TRIEBEL [53] and [54] for a normed space $X$ we denote by $\|x \mid X\|$ the norm of the vector $x \in X$. All unimportant positive constants are denoted with $c$, sometimes with additional subscripts within the same formula.

\section{Function spaces on some subsets of $\mathbb{R}^{n}$}

\subsection{Some classes of subsets of $\mathbb{R}^{n}: d$-sets, domains with minimally smooth boundary, and $(\varepsilon, \delta)$ domains}

We start by introducing some notions related to the smoothness of a domain in $\mathbb{R}^{n}$.

\subsection{1. $\quad d$-sets}

Let $\mathcal{H}^{d}$ be the $d$-dimensional Hausdorff measure. A Borel set $\Gamma$ in $\mathbb{R}^{n}$ is called a $d$-set, $0<d \leq n$, see [40, Chapter II] and [55, Definition 3.1], if there exist positive constants $c_{1}$ and $c_{2}$ such that

$$
c_{1} r^{d} \leq \mathcal{H}^{d}(\Gamma \cap B(x, r)) \leq c_{2} r^{d} \text { for } x \in \Gamma, 0<r \leq 1 .
$$

The notion $d$-set occurs both in the theory of function spaces and in fractal geometry, see [40], [55], [22], [23].

Clearly $\mathbb{R}^{n}$ is a $d$-set with $d=n$ and any convex compact set in $\mathbb{R}^{n}$ with nonempty interior is a $d$-set with $d=n$.

Geometrically self-similar sets are typical examples of $d$-sets. In particular the Cantor set in $\mathbb{R}$ and von Koch's snowflake curve in $\mathbb{R}^{2}$ (see for example [24, page xiii] for a picture) are $d$-sets with $d=\log 2 / \log 3$ and $d=\log 4 / \log 3$, respectively.

\subsubsection{Domains with minimally smooth boundary}

Recall that according to [51, page 189] we say that the boundary $\partial \Omega$ of an open subset $\Omega$ of $\mathbb{R}^{n}$ is minimally smooth if there exist:

an $r>0$, an integer $N$, a number $M>0$ and a sequence (finite or infinite) $U_{1}, U_{2}$, ... of open sets such that:

$(\alpha)$ if $x \in \partial \Omega$ then $B(x, r) \subset U_{i}$ for some $i$;

$(\beta)$ no point of $\mathbb{R}^{n}$ is contained in more than $N$ of the sets $U_{i}$; 
$(\gamma)$ for each $i$ there exists an $G_{i}$ with $U_{i} \cap \Omega=U_{i} \cap G_{i}$ where $G_{i}$ is the rotation of a Lipschitz domain of points in $\mathbb{R}^{n}$ of the form

$$
\left\{x=\left(x^{\prime}, t\right): t>\Phi\left(x^{\prime}\right), x^{\prime} \in \mathbb{R}^{n-1}, t \in \mathbb{R}\right\}
$$

where $\phi: \mathbb{R}^{n-1} \rightarrow \mathbb{R}$ is a function (which may depend on $G_{i}$ ) satisfying a HölderLipschitz condition with bound $M$.

Remark 2.1. If $\Omega$ is an open subset of $\mathbb{R}^{n}$ having minimally smooth boundary $\partial \Omega$ then $\partial \Omega$ is a $d$-set with $d=n-1$ and the closure of $\Omega$ is a $d$-set with $d=n$, see [40, Example 3, page 30].

\subsection{3. $\quad(\varepsilon, \delta)$ domains}

An open connected subset $\Omega$ of $\mathbb{R}^{n}$ is an $(\varepsilon, \delta)$ domain, $\varepsilon>0,0<\delta \leq \infty$, note that $\delta=\infty$ is allowed, see [38], if whenever $x, y \in \Omega$ and $|x-y|<\delta$ there is a rectifiable arc $\gamma \subset \Omega$ with length $l(\gamma)$ joining $x$ to $y$ and satisfying:

$$
\begin{gathered}
l(\gamma) \leq \frac{|x-y|}{\varepsilon}, \\
\operatorname{dist}(z, \partial \Omega) \geq \frac{\varepsilon|x-z| \cdot|y-z|}{|x-y|} \text { for all } z \in \gamma .
\end{gathered}
$$

Any Lipschitz domain $\Omega$ is an $(\varepsilon, \delta)$ domain and also an $n$-set, see [60, Example 1]. If, for $n=2$, we add an ingoing cusp to $\Omega$ then $\Omega$ is still an $n$-set but not an $(\varepsilon, \delta)$ domain, and if we instead add an outgoing cusp to $\Omega$ then $\Omega$ is not even an $n$-set.

If $\Omega$ is von Koch's snowflake domain then as remarked in [38, page 73] a straightforward argument shows that $\Omega$ is an $(\varepsilon, \delta)$ domain.

If $\Omega$ is an $(\varepsilon, \delta)$ domain then $|\partial \Omega|=0$, where $|\partial \Omega|$ denotes the Lebesgue measure of the boundary. The boundary of an $(\varepsilon, \delta)$ domain can, however be highly nonrectifiable and, in general, no regularity condition on $\partial \Omega$ can be inferred from the $(\varepsilon, \delta)$ property.

The situation is even worse than the example of the snowflake domain shows. If $n-1 \leq d<n$ one can construct a domain $\Omega \subset \mathbb{R}^{n}$ such that $\Omega$ is an $(\varepsilon(d), \infty)$ domain and $\mathcal{H}^{d}(U \cap \partial \Omega)>0$ for all open sets $U$ satisfying $U \cap \partial \Omega \neq 0$, see [38, page 73].

Moreover, we would like to point out that in general $(\epsilon, \delta)$ domains are not sets of finite perimeter.

Let us recall briefly the basic facts concerning sets of finite perimeter (Cacciopoli sets). These sets possess an exterior normal which is defined in the same spirit as Lebesgue points of $L_{p}$-derivatives and they are characterized, roughly speaking, by the fact that the Gauss-Green theorem holds.

Let $E \subset \mathbb{R}^{n}$ be a Borel set and $G$ be an open set in $\mathbb{R}^{n}$. The perimeter (cf. [62, Definition 5.4.1]) of $E$ in $G$ is defined as

$$
P(E, G)=\sup \left\{\int_{E} \operatorname{div} g(x) d x: g=\left(g_{1}, \ldots, g_{n}\right) \in C_{0}^{1}\left(G, \mathbb{R}^{n}\right),|g(x)| \leq 1\right\} .
$$


If $G=\mathbb{R}^{n}$ let $P(E)=P\left(E, \mathbb{R}^{n}\right)$. The set $E$ is said to be of locally finite perimeter (or Cacciopoli set, see [32, Definition 1.6]) if $P(E, G)<\infty$ for any bounded open set $G$. If $E$ is of finite perimeter in $\mathbb{R}^{n}$ then $E$ is simply called a set of finite perimeter.

The theory of sets of finite perimeter was initiated by R. CACCIOpOLI in [13] and E. De GIORGI in [15] and [16] and subsequently developed by many contributors, see [62, page 281], as well as [43, §6.7, page 341] for a list of references.

Note that if $\Omega$ is a bounded domain with $C^{2}$ boundary, or if $\Omega$ is minimally smooth, or if $\Omega$ has a Lipschitz boundary then it has locally finite perimeter, see for example [62, Remark 5.4.2] and [20, page 209].

Remark 2.2. The classical snowflake domain in $\mathbb{R}^{2}$ has not locally finite perimeter; thus $(\varepsilon, \delta)$ domains have not finite perimeter in general.

We were not able to find a reference so we will give a proof of this fact.

Proof. We start recalling that if $E$ is a set with locally finite perimeter then a point $x$ is said to belong to the reduced boundary $\partial^{-} E$ of $E$ (see [62, Definition 5.5.1] and [32, Definition 3.3]) if:

(i) $P(E, B(x, r))>0$ for all $r>0$;

(ii) the limit $\nu(x)=\lim _{r \rightarrow 0} \nu_{r}(x)$ exists, where

$$
\nu_{r}(x)=\frac{1}{P(E, B(x, r))} \int_{B(x, r)} D \chi_{E}
$$

and $|\nu(x)|=1$ ( $\chi_{E}$ is the characteristic function of $\left.E\right)$.

Note that if $\partial E$ is a $C^{1}$ hypersurface then $\partial^{-} E=\partial E$ and $\nu$ is the unit inner normal to $E$ at $x$, see [32, Example 3.4].

The notation $\partial^{-} E$ for the reduced boundary is taken from [62, Definition 5.5.1] and the notation $\partial^{-}$in $\partial^{-} E$ is used to indicate that the normal to $E$ is pointing in the direction opposite to the gradient.

Now we return to our proof.

If we would assume that the snowflake domain (let us denote it with $E$ ) has locally finite perimeter then, according to [62, Theorem 5.7.3], its reduced boundary $\partial^{-} E$ is countably $(n-1)$ rectifiable, i. e.

$$
\partial^{-} E \subset E_{0} \cup\left(\bigcup_{i=1}^{\infty} f_{i}\left(\mathbb{R}^{n}\right)\right)
$$

where $\mathcal{H}^{n-1}\left(E_{0}\right)=0$ and each $f_{i}: \mathbb{R}^{n-1} \rightarrow \mathbb{R}^{n}$ is Lipschitz.

We know from [38, page 73] that any subarc of the boundary is non-rectifiable. Thus the reduced boundary of the snowflake domain is empty.

But this last fact contradicts [32, Theorem 4.4.] which states that the reduced boundary $\partial^{-} E$ of a set with locally finite perimeter is dense in $\partial E$.

Thus the snowflake domain has not locally finite perimeter. 


\subsection{Preliminaries on function spaces on domains}

Let $\Omega$ be a domain in the Euclidean $n$-space $\mathbb{R}^{n}$ and let $\mathcal{D}^{\prime}(\Omega)$ be the collection of all distributions on $\Omega$.

Let $k \in \mathbb{N}$ and $1<p<\infty$. The Sobolev space $W_{p}^{k}(\Omega)$ is defined as the collection of all $u \in L_{p}(\Omega)$ such that

$$
\left\|u \mid W_{p}^{k}(\Omega)\right\|=\left(\sum_{|\alpha| \leq k}\left\|D^{\alpha} u \mid L_{p}(\Omega)\right\|^{p}\right)^{1 / p}<\infty .
$$

Of course $W_{p}^{k}(\Omega)$ is considered as a subspace of $\mathcal{D}^{\prime}(\Omega)$, in particular the derivatives $D^{\alpha} u$ must be understood in the sense of distributions.

For any $k \in \mathbb{N}$ and $1<p<\infty$ the Sobolev space $W_{p}^{k}(\Omega)$ is a Banach space (Hilbert space for $p=2)$.

Formula (2.1) is called an inner description of the space $W_{p}^{k}(\Omega)$ because only points $x \in \Omega$ are involved in the definition.

If $\Omega$ is a bounded domain with smooth boundary then $W_{p}^{k}(\Omega)$ coincides with the restriction of $W_{p}^{k}\left(\mathbb{R}^{n}\right)=F_{p 2}^{k}\left(\mathbb{R}^{n}\right)$ to $\Omega$, where $F_{p 2}^{k}\left(\mathbb{R}^{n}\right)$ is a Triebel-Lizorkin space, i. e.

$$
W_{p}^{k}(\Omega)=\left\{u \in \mathcal{D}^{\prime}(\Omega): \exists v \in W_{p}^{k}\left(\mathbb{R}^{n}\right) \text { with } v \mid \Omega=u\right\},
$$

where $v \mid \Omega$ means the restriction of $v$ to $\Omega$ in the sense of $\mathcal{D}^{\prime}(\Omega)$. Moreover,

$$
\inf \left\|v \mid W_{p}^{k}\left(\mathbb{R}^{n}\right)\right\|
$$

where the infimum is taken over all $v \in W_{p}^{k}\left(\mathbb{R}^{n}\right)$ with $v \mid \Omega=u$ is an equivalent norm on $W_{p}^{k}(\Omega)$.

For Sobolev spaces the above situation is quite satisfactory if $\Omega$ is a bounded domain with smooth boundary (it is well-known that rather weak assumptions on the boundary of the bounded domain $\Omega$ are sufficient in order to prove that $(2.1)$ on the one hand and (2.2) and (2.3) on the other hand are equivalent to each other, see [53, Subsection 4.2.3]).

The situation becomes more complicated if one replaces $W_{p}^{k}$ in (2.2) and (2.3) by Sobolev spaces of fractional order or, more generally, by $B_{p q}^{s}$ and $F_{p q}^{s}$ spaces, i. e. Besov and Triebel-Lizorkin spaces. It turns out that the problem of inner descriptions of function spaces on domains is closely related with the problem of the existence of an extension operator.

Recall that if $s \in \mathbb{R}$ and $1<p<\infty$ then the fractional Sobolev space (on $\mathbb{R}^{n}$ ) $H_{p}^{s}\left(\mathbb{R}^{n}\right)$ is defined as the collection of all $u \in \mathcal{S}^{\prime}\left(\mathbb{R}^{n}\right)$ such that

$$
\left\|u\left|H_{p}^{s}\left(\mathbb{R}^{n}\right)\|=\|\left(\left(1+|\cdot|^{2}\right)^{s / 2} \widehat{u}\right)^{\vee}\right| L_{p}\left(\mathbb{R}^{n}\right)\right\|<\infty
$$

where $\widehat{u}$ and $v^{\vee}$ denote the Fourier and inverse Fourier transform of the tempered distributions $u$ and $v$, respectively.

These spaces are also called by some authors Liouville spaces (mainly in the Russian literature) or Bessel potential spaces (see for example [51] and [40]). 
If $\Omega$ is now a domain in $\mathbb{R}^{n}$ and $s \in \mathbb{R}$ then $H_{p}^{s}(\Omega)=\left\{v \mid \Omega: v \in H_{p}^{s}\left(\mathbb{R}^{n}\right)\right\}$ is normed by

$$
\left\|u \mid H_{p}^{s}(\Omega)\right\|=\inf \left\{\left\|v\left|H_{p}^{s}\left(\mathbb{R}^{n}\right) \|: v\right| \Omega=u \operatorname{in} \mathcal{D}^{\prime}(\Omega)\right\} .\right.
$$

These spaces are Banach spaces and for $p=2$ we set $H^{s}(\Omega)=H_{2}^{s}(\Omega)$ and $H^{s}(\Omega)$ becomes a Hilbert space in a natural way.

The theory of $H_{p}^{s}\left(\mathbb{R}^{n}\right)$ and $H_{p}^{s}(\Omega)$ is part of the more general theory of spaces $F_{p q}^{s}\left(\mathbb{R}^{n}\right)$ and $F_{p q}^{s}(\Omega)(s \in \mathbb{R}, 0<p<\infty, 0<q \leq \infty)$ since $F_{p 2}^{s}=H_{p}^{s}$ for $s \in \mathbb{R}$ and $1<p<\infty$. The reader can find a detailed treatment of the theory of these spaces in the monographs of H. Triebel, [53] and [54]. For a more recent account on the theory one can see also [46] and [19].

The spaces $H_{p}^{s}\left(\mathbb{R}^{n}\right)$ are generalizations of $W_{p}^{k}\left(\mathbb{R}^{n}\right)$ in the following sense: if $k \in \mathbb{N}$ and $1<p<\infty$ then $H_{p}^{k}\left(\mathbb{R}^{n}\right)=W_{p}^{k}\left(\mathbb{R}^{n}\right)$, see for example [51, page 135] and [54, Theorem 1.3.2/1].

\subsection{Sobolev spaces on $(\varepsilon, \delta)$ domains}

The $(\varepsilon, \delta)$ domains were introduced by P. W. Jones in [38]. He proved that if $\Omega$ is an $(\varepsilon, \delta)$ domain in $\mathbb{R}^{n}$ then there is an extension operator from the Sobolev space $W_{p}^{k}(\Omega), k \in \mathbb{N}, 1<p<\infty$ to $W_{p}^{k}\left(\mathbb{R}^{n}\right)$ (the extension operators are different for different values of $k$ ).

Moreover, the extension operator may be chosen as a linear operator with norm depending only on $\varepsilon, \delta, p, k$ and $n$. This result extends some earlier results of E. M. STEIN, see [51, page $180-192]$ concerning the existence of linear and bounded extension operators for Sobolev spaces on Lipschitz domains or on domains having minimally smooth boundary.

P.W. JONEs showed that his result is in some sense best possible: if $n=2$, if $\Omega$ is finitely connected and if for $1 \leq p \leq \infty$ and $k \in \mathbb{N}_{0}$ there is a bounded linear extension operator $W_{p}^{k}(\Omega) \rightarrow W_{p}^{k}\left(\mathbb{R}^{n}\right)$ then $\Omega$ is an $(\varepsilon, \delta)$ domain for some $\varepsilon, \delta>0$. The reader is referred to V. G. MAZ'JA and S. V. PoBorChI, see [44, page $44-46$ ], for a list of results related to the theorem of P. W. JonEs.

In [37] D. Jerison and C. Kenig have shown that a large number of potential theoretic properties, known to be true for Lipschitz domains, remain valid for $(\varepsilon, \delta)$ domains. In some sense $(\varepsilon, \delta)$ domains are the worst domains on which the classical function spaces have similar properties to those on the Euclidean upper half space.

Extension operators for anisotropic Sobolev spaces on $(\varepsilon, \delta)$ domains were constructed by B. L. FAJN in [21], extension operators for Besov spaces on $(\varepsilon, \delta)$ domains were constructed by R. A. DeVore and R. C. Sharpley in [17], extension operators for Triebel-Lizorkin spaces (even in the anisotropic context) were constructed by A. SeEger in [49].

In what follows we will need only a weak version of A. SEEGER's result and it is stated below. However the reader interested in the topic of extension operators for Triebel-Lizorkin spaces on $(\varepsilon, \delta)$ domains can consult the work [49] for details, proofs, and further references. 
Theorem 2.3. ([49, Theorem 2].) Let $\Omega$ be an $(\varepsilon, \delta)$ domain and $s>0,1<p<\infty$. Then there exists a bounded linear operator $E: H_{p}^{s}(\Omega) \rightarrow H_{p}^{s}\left(\mathbb{R}^{n}\right)$ such that $E u=u$ almost everywhere on $\Omega$ for all $u \in H_{p}^{s}(\Omega)$.

Clearly this is an extension of P. W. Jones' result. In particular, in [49, Corollaries 1,2$]$ the author obtained characterizations for the norm in $H_{p}^{s}(\Omega)$ in terms of oscillations and differences. We will use the following theorem.

Theorem 2.4. ([49, Corollary 2].) Let $\Omega$ be an $(\varepsilon, \delta)$ domain, $0<s<1$ and $1<p<\infty$. Then

$$
\left\|u\left|L_{p}(\Omega)\|+\|\left(\int_{0}^{\rho(\cdot)}\left(\frac{1}{\left|V^{1}(\cdot, t)\right|} \int_{V^{1}(\cdot, t)}\left|\Delta_{h} u(\cdot)\right| d h\right)^{2} \frac{d t}{t^{1+2 s}}\right)^{1 / 2}\right| L_{p}(\Omega)\right\|
$$

is an equivalent norm in $H_{p}^{s}(\Omega)$.

Here $V^{1}(x, t)=\left\{h \in \mathbb{R}^{n}:|h| \leq t\right.$ and $x+\tau h \in \Omega$ for all $\left.-1 \leq \tau \leq 1\right\}$, cf. also [54, Subsections 3.5.2, 5.2.2], $\left|V^{1}(x, t)\right|$ denotes its Lebesgue measure,

$$
\rho(x)=\frac{1}{2} \operatorname{dist}(x, \partial \Omega) \text { for any } x \in \Omega \text {, }
$$

and

$$
\Delta_{h} u(x)=u(x+h)-u(x)
$$

are the first order differences with step $h$.

Note that if one considers $s \geq 1$ then higher order differences have to be taken in (2.5) to get equivalent norms in $H_{p}^{s}(\Omega)$.

\subsection{Sobolev spaces on $d$-sets}

If $\Omega$ is a bounded smooth domain in $\mathbb{R}^{n}$ and $s \in \mathbb{R}, 0<p \leq \infty, 0<q \leq \infty$, then one can introduce Besov spaces $B_{p q}^{s}(\Gamma)$ or (for $\left.1 \leq p<\infty\right)$ Sobolev spaces $H_{p}^{s}(\Gamma)$ on the compact $(n-1)$ dimensional manifold $\Gamma=\partial \Omega$. For this purpose one needs pointwise multipliers and diffeomorphism properties of the related spaces on $\mathbb{R}^{n}$. The basic idea is to reduce spaces on $\Gamma$ via an atlas of finitely many local $C^{\infty}$ charts to corresponding spaces on $\mathbb{R}^{n-1}$. In that case we do not want to go into details and refer to [53, Subsection 3.2.2].

The situation is essentially different if $\Gamma=\partial \Omega$ is non smooth. Let $\Gamma$ be a closed set in $\mathbb{R}^{n}$ with Lebesgue measure $|\Gamma|=0$. It is of interest to define intrinsically spaces of Hölder (Lipschitz) type or, more general of $B_{p q}^{s}$ and $F_{p q}^{s}$ and Hardy type, and to ask for (linear and) bounded extension operators from these spaces into suitable spaces on $\mathbb{R}^{n}$. This theory began in the thirties with Whitney's construction of a linear and bounded extension operator on intrinsically defined Hölder spaces on $\Gamma$ into the corresponding spaces on $\mathbb{R}^{n}$, see [51, Chapter 6] for details. Extending this procedure, A. Jonsson and H. WALLIN studied more general spaces, especially Besov spaces but also Hardy spaces on $\Gamma$ in [40]. 
A detailed discussion including many references on various ways of defining function spaces on fractals, as well as a new approach on the construction of Besov spaces on $d$-sets, may be found in [55, Chapter 20] and will not be repeated here.

Here we will follow mainly [40].

Let $\Gamma$ be a $d$-set in $\mathbb{R}^{n}$ with $0<d<n$. If $1 \leq p<\infty$ then $L_{p}(\Gamma)$ has the usual meaning with respect to $\mu=\mathcal{H}^{d} \mid \Gamma$.

Definition 2.5. ([40, Chapter V, page 103].) Let $0<\sigma<1$ and $1 \leq p<\infty$. Let $\Gamma$ be a $d$-set in $\mathbb{R}^{n}, 0<d<n$. The Besov space $B_{p p}^{\sigma}(\Gamma)$ on $\Gamma$ is the collection of all functions $u$ defined on $\Gamma$ such that

$$
\left\|u\left|B_{p p}^{\sigma}(\Gamma)\|=\| u\right| L_{p}(\Gamma)\right\|+\left(\iint_{|x-y|<1} \frac{|u(x)-u(y)|^{p}}{|x-y|^{d+\sigma p}} d \mu(x) d \mu(y)\right)^{1 / p}<\infty .
$$

For equivalent norms in $B_{p p}^{\sigma}(\Gamma)$ one can see [40, page 108], [41, Definition 1.4] or [39].

In the rest of the paper we do not deal with Besov spaces of higher order of smoothness $\sigma$ on a $d$-set $\Gamma$. The reader should note that if $\sigma \geq 1$ the definition of $B_{p p}^{\sigma}(\Gamma)$ is more complicated, see [40, Chapter V] for details.

Let us remark that if $p=2$ then $H^{\sigma}(\Gamma)=B_{22}^{\sigma}(\Gamma)$ becomes a Hilbert space in a canonical way.

We will use a characterization of Besov spaces on $d$-sets as traces of appropriate Sobolev spaces on $\mathbb{R}^{n}$.

Let again $\Gamma$ be a $d$-set in $\mathbb{R}^{n}, 0<d<n$. If the function $u$ is defined and continuous on $\mathbb{R}^{n}$ or has a continuous representative if $u$ is an $L_{p}$ function, then the restriction or trace of $u$ to $\Gamma$ is defined pointwise and denoted $u \mid \Gamma$. If $\Gamma$ has positive $n$-dimensional Lebesgue measure and we consider functions $u$ defined almost everywhere in $\mathbb{R}^{n}$, then the pointwise restriction to $\Gamma$ gives functions defined almost everywhere on $\Gamma$.

The restriction of $u$ to $\Gamma$ may, however, be defined in a natural way also in many situations when $\Gamma$ has $n$-dimensional Lebesgue measure zero and $u$ is defined almost everywhere in $\mathbb{R}^{n}$. There are several ways to do this; here we mention one possibility which we shall use throughout.

We say $u \in L_{1, l o c}\left(\mathbb{R}^{n}\right)$ can be strictly defined at the point $x$ if the limit

$$
\bar{u}(x)=\lim _{r \rightarrow 0} \frac{1}{|B(x, r)|} \int_{B(x, r)} u(y) d y \quad \text { exists. }
$$

By the differentiation theorem of Lebesgue we have $u=\bar{u}$ almost everywhere in $\mathbb{R}^{n}$, see for example [62, Subsection 1.3.8] for a proof. By redefining, if necessary, $u$ on a set of $n$-dimensional Lebesgue measure zero, we can thus obtain $u=\bar{u}$ at all points where the limit $\bar{u}$ exists. When this is done we say that $u$ is strictly defined and make the following definition of $u \mid \Gamma$.

Definition 2.6. If $u \in L_{1, l o c}\left(\mathbb{R}^{n}\right)$ and $\Gamma \subset \mathbb{R}^{n}$ then $u \mid \Gamma$ is the pointwise restriction to $\Gamma$ of the strictly defined function $u$. 
The next result is taken from [40, Chapter VII] but one should consult also [55, Section 20].

Theorem 2.7. Let $0<d<n$ and let $\Gamma$ be a $d$-set in $\mathbb{R}^{n}$. Let $1<p<\infty$ and let $s \in \mathbb{R}$ such that

$$
\frac{n-d}{p}<s<1+\frac{n-d}{p} .
$$

Then the trace operator $\operatorname{Tr}_{\Gamma}: u \mapsto u \mid \Gamma$ defined by means of Definition 2.6 is a bounded linear surjection

$$
\operatorname{Tr}_{\Gamma}: H_{p}^{s}\left(\mathbb{R}^{n}\right) \longrightarrow B_{p p}^{s-\frac{n-d}{p}}(\Gamma)
$$

with a bounded linear right inverse $E_{\Gamma}$ (the extension operator).

In the rest of the paper we will not deal with the limiting case $s=\frac{n-d}{p}$. However the interested reader should consult Theorems 2 and 3 in [58] and [55, Theorems 18.2, 18.6] for this situation.

\section{Traces on the boundary of Sobolev spaces on $(\varepsilon, \delta)$ do- mains}

Let $\Omega$ be a domain in $\mathbb{R}^{n}$. We say $u \in L_{1, l o c}(\Omega)$ can be strictly defined at the point $x \in \Omega \cup \partial \Omega$ if the limit

$$
\widetilde{u}(x):=\lim _{r \rightarrow 0} \frac{1}{|B(x, r) \cap \Omega|} \int_{B(x, r) \cap \Omega} u(y) d y
$$

exists. If we replace $\Omega$ by $\mathbb{R}^{n}$ we get the same definition as in (2.7).

Definition 3.1. The trace $\operatorname{tr}_{\partial \Omega} u$ of a function $u \in L_{1, l o c}(\Omega)$ is defined as the function $(u \mid \partial \Omega)(x)=\widetilde{u}(x)$ at every point $x \in \partial \Omega$ where $\widetilde{u}(x)$ exists.

This definition of the trace and the notation $u \mid \Gamma$ is consistent with the definition and notation based on (2.7) in the following sense.

Theorem 3.2. Let $n-1 \leq d<n$ and let $\Omega$ be a bounded $(\varepsilon, \delta)$ domain in $\mathbb{R}^{n}$ such that $\partial \Omega=\Gamma$ is a d-set. Let $1<p<\infty$ and $s \in \mathbb{R}$ such that

$$
\frac{n-d}{p}<s<1+\frac{n-d}{p}
$$

Assume $u \in H_{p}^{s}(\Omega)$ and let Eu be any function in $H_{p}^{s}\left(\mathbb{R}^{n}\right)$ such that $E u=u$ almost everywhere in $\Omega$, cf. Theorem 2.3 .

Then $\widetilde{u}$ and $\overline{E u}$ exist and coincide on $\Gamma$, with a possible exception on a set which has $d$-dimensional Hausdorff measure zero. 
The proof of this theorem depends on continuity properties of Bessel potentials and can be achieved line by line from the proof of Theorem 1 in [60], which in turn was based on [40, page 209]. We do not go into details.

Notation. If extra clarity is desirable we adopt the convention of denoting the trace on a $d$-set $\Gamma=\partial \Omega$ of a function defined on $\mathbb{R}^{n}$ by $\operatorname{Tr}_{\Gamma}$ and the trace of a function defined only on $\Omega$ by $\operatorname{tr}_{\Gamma}$.

Theorem 3.3. Let $n-1 \leq d<n$ and let $\Omega$ be a bounded $(\varepsilon, \delta)$ domain in $\mathbb{R}^{n}$ such that $\partial \Omega=\Gamma$ is a $d$-set. Let $1<p<\infty$ and $s \in \mathbb{R}$ such that

$$
\frac{n-d}{p}<s<1+\frac{n-d}{p}
$$

Then the trace operator $\operatorname{tr}_{\partial \Omega}: u \mapsto u \mid \partial \Omega$ defined by means of Definition 3.1 is a bounded linear surjection

$$
\operatorname{tr}_{\partial \Omega}: H_{p}^{s}(\Omega) \longrightarrow B_{p p}^{s-\frac{n-d}{p}}(\partial \Omega) .
$$

Moreover, there exists a linear bounded right inverse $\widetilde{\gamma}$.

Remark 3.4. This result extends [41, Theorem 6] and [60, Theorem 2] where Sobolev spaces $W_{p}^{k}(\Omega)$ of integer order $k \geq 1$ of smoothness on $(\varepsilon, \delta)$ domains are considered.

Proof of Theorem 3.3. The method is that from [40, Theorem 1, page 208], cf. also [60, Theorem 2]. We have the following embeddings

$$
H_{p}^{s}(\Omega) \stackrel{E}{\longrightarrow} H_{p}^{s}\left(\mathbb{R}^{n}\right) \stackrel{\operatorname{Tr}_{\Gamma}}{\longrightarrow} B_{p p}^{s-\frac{n-d}{p}}(\Gamma)
$$

where $E$ is the extension operator from Theorem 2.3 and $\operatorname{Tr}_{\Gamma}$ is the trace operator from Theorem 2.7. We have also

$$
B_{p p}^{s-\frac{n-d}{p}}(\Gamma) \stackrel{E_{\Gamma}}{\longrightarrow} H_{p}^{s}\left(\mathbb{R}^{n}\right) \stackrel{R}{\longrightarrow} H_{p}^{s}(\Omega)
$$

where $E_{\Gamma}$ is the extension operator from Theorem 2.7 and $R$ is the usual restriction operator.

Different ways to define traces of functions on $\Omega$ and on $\mathbb{R}^{n}$ are used, but this does not matter because of Theorem 3.2.

The theorem is now a simple consequence of the embeddings stated in (3.2) and $(3.3)$.

We determine now the kernel of the trace operator from Theorem 3.3.

Theorem 3.5. Let $n-1 \leq d<n$ and let $\Omega$ be a bounded $(\varepsilon, \delta)$ domain in $\mathbb{R}^{n}$ such that $\partial \Omega=\Gamma$ is a d-set. Let $1<p<\infty$ and $s \in \mathbb{R}$ such that

$$
\frac{n-d}{p}<s \leq 1
$$


Then the kernel of the trace operator

$$
\operatorname{tr}_{\partial \Omega}: H_{p}^{s}(\Omega) \longrightarrow B_{p p}^{s-\frac{n-d}{p}}(\partial \Omega)
$$

is $\stackrel{\circ}{H}_{p}^{s}(\Omega)$, i. e. the closure of $C_{0}^{\infty}(\Omega)$ in the norm of $H_{p}^{s}(\Omega)$.

Remark 3.6. This result is the extension to fractional order Sobolev spaces $H_{p}^{s}(\Omega)$ of a result of H. WALlin, see [60, Theorem 3]. (Our) Theorem 3.5 and Theorem 3 in [60] have as forerunners the work of J. MARSCHALL [42] who established a similar result for Slobodeckij spaces on domains having minimally smooth boundary. Related (or more general) results in this direction may be founded in [57], Proposition 19.5. As far as spectral synthesis is concerned we refer to [57], 19.6.

Remark 3.7. In the proof of Theorem 3.5 we will use the intrinsic characterization of A. SEEgER for the norm in $H_{p}^{s}(\Omega)$ if $s<1$, see Theorem 2.4. Using higher order differences the method may probably extended to prove that the kernel of the trace operator (3.4) is $\stackrel{\circ}{H}_{p}^{s}(\Omega)$ for any $\frac{n-d}{p}<s<1+\frac{n-d}{p}$ or, more generally, satisfying $m+\frac{n-d}{p}<s<m+1+\frac{n-d}{p}$, where $m \in \mathbb{N}$.

One can specialize Theorem 3.5 to smooth domains $\Omega$, or to domains having minimally smooth boundary, taking into account Remark 2.1 .

Corollary 3.8. If $\Omega$ is a minimally smooth bounded domain in $\mathbb{R}^{n}, 1<p<\infty$ and

$$
\frac{1}{p}<s \leq 1
$$

then

$$
\stackrel{\circ}{H}_{p}^{s}(\Omega)=\left\{u \in H_{p}^{s}(\Omega): \operatorname{tr}_{\partial \Omega} u=0\right\}
$$

At least for smooth domains this result is well-known for $\frac{1}{p}<s<1+\frac{n-d}{p}$, see for example [52, Theorem 4.7.1].

Pr o of of Theorem 3.5. Since for $s=1$ the theorem was proved by H. WALLIN in [60] we may assume, without loss of generality $s<1$.

Step 1. In this first step we follow the technique from [60, Theorem 3] which in fact was based on [42, Theorem 1].

Clearly $\stackrel{\circ}{H}_{p}^{s}(\Omega)$ is a subset of the kernel of $\operatorname{tr}_{\partial \Omega}$ so we concentrate on proving the reverse inclusion.

Let $f \in H_{p}^{s}(\Omega)$ with $\operatorname{tr}_{\partial \Omega} f=0$ in $B_{p p}^{s-\frac{n-d}{p}}(\partial \Omega)$. By Theorem 2.3 we may extend $f$ to a function $F$ in $H_{p}^{s}\left(\mathbb{R}^{n}\right)$ and let us remark that $\operatorname{Tr}_{\partial \Omega} F=0$ by Theorem 3.2.

Let $F_{j} \in C_{0}^{\infty}\left(\mathbb{R}^{n}\right)$ be a sequence such that $F_{j} \rightarrow F$ in $H_{p}^{s}\left(\mathbb{R}^{n}\right)$; it is well-known that such a sequence exists, see for example [3, Theorem 7.63]. Using the boundedness of the operator $\operatorname{Tr}_{\partial \Omega}$ from Theorem 2.7 we get $\operatorname{Tr}_{\partial \Omega} F_{j} \rightarrow \operatorname{Tr}_{\partial \Omega} F=0$ in $B_{p p}^{s-\frac{n-d}{p}}(\partial \Omega)$. 
Let $E_{\partial \Omega}$ be the bounded extension operator from Theorem 2.7. Defining

$$
G_{j}=E_{\partial \Omega}\left(\operatorname{Tr}_{\partial \Omega} F_{j}\right) \in H_{p}^{s}\left(\mathbb{R}^{n}\right)
$$

let us remark that $G_{j} \rightarrow 0$ in $H_{p}^{s}\left(\mathbb{R}^{n}\right)$ by the boundedness of $E_{\partial \Omega}$.

The extension operator $E_{\partial \Omega}$ is of Whitney type, see [40, page 199 and 157]. It may be checked in the proof of Whitney's extension theorem, see [51, page 177], that $\operatorname{supp} G_{j}$ is compact and each $G_{j}$ belongs to the Lipschitz space $\operatorname{Lip}\left(1, \mathbb{R}^{n}\right)$, see $[40$, Theorem VI.3] and [42, Lemma 6].

Recall $u \in \operatorname{Lip}\left(1, \mathbb{R}^{n}\right)$, see $\left[40\right.$, Subsection 1.2], if $u \in C^{0}\left(\mathbb{R}^{n}\right)$ and for some constant $M>0$

$$
|u(x)| \leq M \text { for } \quad x \in \mathbb{R}^{n}
$$

and

$$
\left|\Delta_{h} u(x)\right| \leq M|h| \text { for } \quad x, h \in \mathbb{R}^{n},
$$

where $\Delta_{h}$ denotes, as usual, the first order difference with step $h, \Delta_{h} g(x)=g(x+h)-$ $g(x)$.

These spaces are sometimes denoted $C^{0,1}\left(\overline{\mathbb{R}^{n}}\right)$, see for example [3].

Let now $H_{j}=F_{j}-G_{j} \in H_{p}^{s}\left(\mathbb{R}^{n}\right)$. It is easy to see that for all $j \geq 1 \operatorname{supp} H_{j}$ is compact, $\operatorname{Tr}_{\partial \Omega} H_{j}=0$ in $B_{p p}^{s-\frac{n-d}{p}}(\partial \Omega)$ and $H_{j} \rightarrow F$ in $H_{p}^{s}\left(\mathbb{R}^{n}\right)$ which implies $H_{j} \mid \Omega \rightarrow f$ in $H_{p}^{s}(\Omega)$ (here one has to use the fact that the norm in $H_{p}^{s}(\Omega)$ is initially defined by the restriction procedure).

Hence the proof is reduced to the case when the original function $f \in H_{p}^{s}(\Omega)$ has compact support in $\mathbb{R}^{n}$, belongs to $\operatorname{Ker} \operatorname{tr}_{\partial \Omega}$ but is also in $\operatorname{Lip}\left(1, \mathbb{R}^{n}\right)$, where

$$
\frac{n-d}{p}<s<1
$$

Step 2. Let $s$ as in (3.5) and $f \in H_{p}^{s}(\Omega)$ such that $f \in \operatorname{Ker}_{\partial \Omega} \cap \operatorname{Lip}\left(1, \mathbb{R}^{n}\right), f$ with compact support in $\mathbb{R}^{n}$. Assuming this from now on we observe $f=0$ everywhere on $\partial \Omega$, see [40, Proposition 5 and Theorem 4, page 41]. Hence we get for some constant $c>0$ the inequality

$$
|f(x)| \leq c \operatorname{dist}(x, \partial \Omega) \text { for all } x .
$$

Given $\varepsilon>0$, take $\varphi_{\varepsilon} \in C_{0}^{\infty}(\Omega)$ such that

$$
\varphi_{\varepsilon}(x)=1 \text { for all } x \in \Omega \text { with } \operatorname{dist}(x, \partial \Omega) \geq \varepsilon
$$

and such that for all $\alpha \in \mathbb{N}_{0}^{n}$ there is a constant $c_{\alpha}>0$

$$
\left|D^{\alpha} \varphi_{\varepsilon}(x)\right| \leq c_{\alpha} \varepsilon^{-|\alpha|}, \text { for all } x \in \mathbb{R}^{n},
$$

see [42]. Define $f_{\varepsilon}=\varphi_{\varepsilon} \cdot f$.

Then clearly $\operatorname{supp} f_{\varepsilon}$ is a compact subset of $\Omega$ and we have $f_{\varepsilon} \rightarrow f$ in $H_{p}^{s}(\Omega)$ as $\varepsilon \rightarrow 0$. 
To verify our last claim let us first note that clearly

$$
\lim _{\varepsilon \rightarrow 0}\left\|f_{\varepsilon}-f \mid L_{p}(\Omega)\right\|=0
$$

For any $g$ let us denote now

$$
v(g, x)=\left(\int_{0}^{\rho(x)}\left(\frac{1}{\left|V^{1}(x, t)\right|} \int_{V^{1}(x, t)}\left|\Delta_{h} g(x)\right| d h\right)^{2} \frac{d t}{t^{1+2 s}}\right)^{1 / 2},
$$

see (2.5). According to Theorem 2.4 it remains to prove

$$
\lim _{\varepsilon \rightarrow 0}\left\|v\left(f_{\varepsilon}-f, \cdot\right)\left|L_{p}(\Omega)\left\|=\lim _{\varepsilon \rightarrow 0}\right\| v\left(\psi_{\varepsilon} f, \cdot\right)\right| L_{p}(\Omega)\right\|=0
$$

where we have denoted $\psi_{\varepsilon}=1-\varphi_{\varepsilon}$.

Now $\Delta_{h}\left(\psi_{\varepsilon} f\right)(x)=\left(\psi_{\varepsilon} f\right)(x+h)-\left(\psi_{\varepsilon} f\right)(x)=\psi_{\varepsilon}(x)\left(\Delta_{h} f\right)(x)+f(x+h)\left(\Delta_{h} \psi_{\varepsilon}\right)(x)$ and consequently there exists a constant $c>0$ such that

where

$$
\left|v\left(\psi_{\varepsilon} f, \cdot\right)\right| \leq c\left(I_{1, \varepsilon}(\cdot)+I_{2, \varepsilon}(\cdot)\right)
$$

$$
\begin{aligned}
I_{1, \varepsilon}(x) & =\left(\int_{0}^{\rho(x)}\left(\frac{1}{\left|V^{1}(x, t)\right|} \int_{V^{1}(x, t)}\left|\psi_{\varepsilon}(x)\right| \cdot\left|\left(\Delta_{h} f\right)(x)\right| d h\right)^{2} \frac{d t}{t^{1+2 s}}\right)^{1 / 2} \\
& =\left|\psi_{\varepsilon}(x)\right| \cdot v(f, x)
\end{aligned}
$$

and

$$
I_{2, \varepsilon}(x)=\left(\int_{0}^{\rho(x)}\left(\frac{1}{\left|V^{1}(x, t)\right|} \int_{V^{1}(x, t)}|f(x+h)| \cdot\left|\left(\Delta_{h} \psi_{\varepsilon}\right)(x)\right| d h\right)^{2} \frac{d t}{t^{1+2 s}}\right)^{1 / 2} .
$$

Due to the properties of $\varphi_{\varepsilon}$ we have $\psi_{\varepsilon}(\cdot) \rightarrow 0$ pointwise on $\Omega$ for $\varepsilon \rightarrow 0$ and there is a constant $c>0$ (independent of $\varepsilon$ ) such that $\left|\psi_{\varepsilon}(x)\right| \leq c$ for any $x \in \Omega$.

Since $f \in H_{p}^{s}(\Omega)$ implies $v(f, \cdot) \in L_{p}(\Omega)$ we conclude $I_{1, \varepsilon}(\cdot)$ tends pointwise to 0 on $\Omega$ for $\varepsilon \rightarrow 0$ and is uniformly bounded by a function in $L_{p}(\Omega)$.

Using the theorem of Lebesgue (dominated convergence), we get

$$
\left\|I_{1, \varepsilon}(\cdot) \mid L_{p}(\Omega)\right\| \longrightarrow 0 \text { as } \varepsilon \rightarrow 0 \text {. }
$$

Now we will show that $\left\|I_{2, \varepsilon}(\cdot) \mid L_{p}(\Omega)\right\| \rightarrow 0$ as $\varepsilon \rightarrow 0$. Remark first that using the assumption (3.7) there exists a constant $c>0$ such that

$$
\left|\left(\Delta_{h} \psi_{\varepsilon}\right)(x)\right| \leq c \frac{|h|}{\varepsilon} \quad \text { for any } \quad x \in \Omega
$$

Let us denote $\Omega_{\varepsilon}=\{x \in \Omega: \operatorname{dist}(x, \partial \Omega)<\varepsilon\}$. Then

$$
\left\|I_{2, \varepsilon}(\cdot)\left|L_{p}(\Omega)\left\|^{p}=\right\| I_{2, \varepsilon}(\cdot)\right| L_{p}\left(\Omega_{\varepsilon}\right)\right\|^{p}+\left\|I_{2, \varepsilon}(\cdot) \mid L_{p}\left(C \Omega_{\varepsilon}\right)\right\|^{p} .
$$


Assume temporary $x \in \Omega_{\varepsilon}$. Then using (3.6) we have

$$
|f(x+h)| \leq c \operatorname{dist}(x+h, \partial \Omega) \leq c(\operatorname{dist}(x, \partial \Omega)+|h|) \leq c(\varepsilon+|h|) .
$$

By the definition of $V^{1}(x, t)$, see Theorem 2.4, combining (3.9) and (3.10) it follows

$$
\frac{1}{\left|V^{1}(x, t)\right|} \int_{V^{1}(x, t)}|f(x+h)| \cdot\left|\left(\Delta_{h} \psi_{\varepsilon}\right)(x)\right| d h \leq c(\varepsilon+t) \frac{t}{\varepsilon}
$$

and this implies (recall $x \in \Omega_{\varepsilon}$ )

$$
\begin{aligned}
\left|I_{2, \varepsilon}(x)\right| & \leq c\left(\int_{0}^{\rho(x)}\left((\varepsilon+t) \frac{t}{\varepsilon}\right)^{2} \frac{d t}{t^{1+2 s}}\right)^{1 / 2} \\
& =c \frac{1}{\varepsilon}\left(\int_{0}^{\rho(x)}(\varepsilon+t)^{2} t^{1-2 s} d t\right)^{1 / 2} \\
& \leq c^{\prime} \frac{1}{\varepsilon}\left(\varepsilon^{2} \rho(x)^{2-2 s}+\varepsilon \rho(x)^{3-2 s}+\rho(x)^{4-2 s}\right)^{1 / 2} \\
& \leq c^{\prime \prime} \frac{1}{\varepsilon} \varepsilon^{2-s} \\
& =c^{\prime \prime} \varepsilon^{1-s}
\end{aligned}
$$

where in the last inequality we used $\rho(x)=\frac{1}{2} \operatorname{dist}(x, \partial \Omega) \leq \frac{1}{2} \varepsilon$, see (2.6).

Since $\Omega$ is bounded we obtain for some constant $c>0$

$$
\left\|I_{2, \varepsilon}(\cdot)\left|L_{p}\left(\Omega_{\varepsilon}\right) \|^{p} \leq c \varepsilon^{(1-s) p}\right| \Omega \mid\right.
$$

and (using $s<1$ ) this implies $\left\|I_{2, \varepsilon}(\cdot) \mid L_{p}\left(\Omega_{\varepsilon}\right)\right\|^{p} \rightarrow 0$ as $\varepsilon \rightarrow 0$.

Assume now $x \in C \Omega_{\varepsilon}$. Then $\psi_{\varepsilon}(x)=0$ and so $\Delta_{h} \psi_{\varepsilon}(x)=\psi_{\varepsilon}(x+h)$ and the integral over $V^{1}(x, t)$ in the expression of $I_{2, \varepsilon}$ is in fact taken over

$$
V^{1}(x, t) \cap\left\{h \in \mathbb{R}^{n}: \operatorname{dist}(x+h, \partial \Omega)<\varepsilon\right\} .
$$

Moreover, there exists a $t_{x}>0$ such that $V^{1}\left(x, t_{x}\right) \subset C \Omega_{\varepsilon}$. Then the integral over $t$ in the expression of $I_{2, \varepsilon}$ is in fact taken over $\left[t_{x}, \rho(x)\right]$.

By (3.6) we have $|f(x+h)| \leq c \varepsilon$ for $x \in C \Omega_{\varepsilon}$ and $h$ such that $x+h \in \Omega_{\varepsilon}$.

Using (3.7) for $\alpha=0$ there exists a constant $c>0$ independent of $\varepsilon$ such that

$$
\begin{aligned}
\left|I_{2, \varepsilon}(x)\right| & \leq c\left(\int_{t_{x}}^{\rho(x)}\left(\frac{1}{\left|V^{1}(x, t)\right|} \int_{V^{1}(x, t)} \varepsilon d h\right)^{2} \frac{d t}{t^{1+2 s}}\right)^{1 / 2} \\
& \leq c^{\prime} \varepsilon\left(\frac{1}{\rho(x)^{2 s}}-\frac{1}{t_{x}^{2 s}}\right)
\end{aligned}
$$

and this shows that in this case $I_{2, \varepsilon} \rightarrow 0$ pointwise for $\varepsilon \rightarrow 0$.

On the other hand, using again $|f(x+h)| \leq c \varepsilon$ for $x \in C \Omega_{\varepsilon}$ and $h$ such that $x+h \in \Omega_{\varepsilon}$ and the estimate (3.9) we have for some constant $c>0$ independent of $\varepsilon$

$$
\frac{1}{\left|V^{1}(x, t)\right|} \int_{V^{1}(x, t)}|f(x+h)| \cdot\left|\Delta_{h} \psi_{\varepsilon}(x)\right| d h \leq c \varepsilon \frac{t}{\varepsilon}=c t
$$


and so

$$
\left|I_{2, \varepsilon}(x)\right| \leq c\left(\int_{0}^{\rho(x)} t^{1-2 s} d t\right)^{1 / 2}=c \rho(x)^{1-s} .
$$

Since $s<1$ and the domain $\Omega$ is bounded, the last inequality means that on $C \Omega_{\varepsilon}$ the function $I_{2, \varepsilon}$ is uniformly bounded by a function in $L_{p}(\Omega)$.

Applying again the theorem of Lebesgue (dominated convergence) it follows $\left\|I_{2, \varepsilon}(\cdot) \mid L_{p}\left(C \Omega_{\varepsilon}\right)\right\| \rightarrow 0$ as $\varepsilon \rightarrow 0$.

Consequently $\left\|v\left(\psi_{\varepsilon} f, \cdot\right) \mid L_{p}(\Omega)\right\| \rightarrow 0$ as $\varepsilon \rightarrow 0$ and this is exactly the claim (3.8).

Step 3. It only remains to approximate $f_{\varepsilon}$ arbitrarily close in $H_{p}^{s}(\Omega)$ by functions in $C_{0}^{\infty}(\Omega)$. The approximating sequence of functions from $C_{0}^{\infty}(\Omega)$ is constructed in a standard manner, using mollifiers. One has to take the convolution of $f_{\varepsilon}$ with a non-negative function in $C_{0}^{\infty}\left(\mathbb{R}^{n}\right)$ with integral 1 and small support and apply the same technique as in Step 2.

The proof is now complete.

The next result clarifies the situation $0<s<\frac{n-d}{p}$.

Theorem 3.9. Let $n-1 \leq d<n$ and let $\Omega$ be a bounded $(\varepsilon, \delta)$ domain in $\mathbb{R}^{n}$ such that $\partial \Omega=\Gamma$ is a d-set. If $1<p<\infty$ and $s \in \mathbb{R}$ such that

$$
0<s<\frac{n-d}{p}
$$

then

$$
H_{p}^{s}(\Omega)=\stackrel{\circ}{H_{p}^{s}}(\Omega)
$$

Proof. The proof follows step by step the technique of H. TRIEBEL from [56, Theorem 2.1.6] so that we will only sketch it.

Since $\mathcal{S}\left(\mathbb{R}^{n}\right)$ is dense in $H_{p}^{s}\left(\mathbb{R}^{n}\right)$ we have $H_{p}^{s}(\Omega)=\stackrel{\circ}{H_{p}^{s}}(\Omega)$ if any $u \in C_{0}^{\infty}\left(\mathbb{R}^{n}\right)$ can be approximated in $H_{p}^{s}\left(\mathbb{R}^{n}\right)$ by functions in

$$
\mathcal{D}_{\Omega}\left(\mathbb{R}^{n}\right)=\left\{g \in C_{0}^{\infty}\left(\mathbb{R}^{n}\right): g(x)=0 \text { near } \partial \Omega\right\} .
$$

By the technique developed in [55, Corollary 13.9] for any fixed $j \in \mathbb{N}$ in $(\partial \Omega)_{j}=$ $\left\{y \in \mathbb{R}^{n}: \operatorname{dist}(y, \partial \Omega)<2^{-j}\right\}$ we have the atomic decomposition

$$
u(x)=\sum_{m} \lambda_{j m} b_{j m}(x), \quad x \in(\partial \Omega)_{j}
$$

where the sum is taken over those balls $B_{j m}=\left\{y \in \mathbb{R}^{n}:\left|y-2^{-j} m\right|<c 2^{-j}\right\}$ having a non-empty intersection with $(\partial \Omega)_{j}$. Atomic decompositions go back to M. FRAZIER and B. JAWERTH, see [25] and [26].

Since $\partial \Omega$ is a $d$-set the number of these balls can be approximated from above by $c 2^{j d}$. Furthermore, we may assume

$$
\left|\lambda_{j m}\right| \leq C 2^{j\left(s-\frac{n}{p}\right)}
$$


where $C=C(u)$ is independent of $j$. This is a consequence of

$$
\left|b_{j m}(x)\right| \leq 2^{-j\left(s-\frac{n}{p}\right)}
$$

since $b_{j m}$ is an $(s, p)$ atom, see [55, Definition 13.3].

Since $H_{p}^{s}\left(\mathbb{R}^{n}\right)=F_{p 2}^{s}\left(\mathbb{R}^{n}\right)$ by Step 2 in the proof of Theorem 2.1.6 in [56] the desired approximation property holds for $F_{p q_{1}}^{s}\left(\mathbb{R}^{n}\right)$ if, and only if, it holds for $F_{p q_{2}}^{s}\left(\mathbb{R}^{n}\right)$. This independence of the index $q$ goes back to the work [26] but we do not go into details here. Now,

$$
\left\|\lambda=\left(\lambda_{j m}\right) \mid f_{p p}\right\|=\left(\sum_{m}\left|\lambda_{j m}\right|^{p}\right)^{1 / p} \leq c 2^{j\left(s-\frac{n}{p}\right)} 2^{j \frac{d}{p}}=c 2^{j\left(s-\frac{n-d}{p}\right)} ;
$$

for the sequence space $f_{p p}$ we used the notation from [55, Definition 13.5]. Since $s<\frac{n-d}{p}$ we obtain the desired approximation of $u \in \mathcal{D}\left(\mathbb{R}^{n}\right)$ in $F_{p p}^{s}\left(\mathbb{R}^{n}\right)$ by functions belonging to $\mathcal{D}_{\Omega}\left(\mathbb{R}^{n}\right)$.

Remark 3.10. After a first version of this paper was ready, A. CAEtano informed us that in his recent work [12] he obtained an improvement of Theorem 3.9 using some weaker restrictions on the domain $\Omega$ and on its boundary $\partial \Omega$.

\section{Some Dirichlet forms and subordinated Dirichlet forms defined on Sobolev spaces on $(\varepsilon, \delta)$ domains}

\subsection{Preliminaries}

All function spaces we will consider from now on are real. Through all this section $\Omega \subset \mathbb{R}^{n}$ is a bounded $(\varepsilon, \delta)$ domain with boundary $\Gamma=\partial \Omega$ which is a $d$-set with $n-1 \leq d<n$. The first order Sobolev space $H^{1}(\Omega)=H_{2}^{1}(\Omega)=W_{2}^{1}(\Omega)$ has the meaning from (2.1), in particular the norm is given by

$$
\left\|u \mid H^{1}(\Omega)\right\|=\left(\left\|u\left|L_{2}(\Omega)\left\|^{2}+\sum_{j=1}^{n}\right\| \frac{\partial u}{\partial x_{j}}\right| L_{2}(\Omega)\right\|^{2}\right)^{1 / 2}
$$

and it is clear that $\left\|\cdot\left|L_{2}(\Omega)\|+\| \nabla(\cdot)\right| L_{2}(\Omega)\right\|$ is an equivalent norm on $H^{1}(\Omega)$.

Let $\left(a_{k l}\right)_{k, l=1, \ldots, n}$ be a family of functions in $C^{\infty}(\bar{\Omega})$ such that $a_{k l}=a_{l k}$ and such that for some $\lambda_{0}>0$

$$
\lambda_{0}^{-1}|\xi|^{2} \leq \sum_{k, l=1}^{n} a_{k l}(x) \xi_{k} \xi_{l} \leq \lambda_{0}|\xi|^{2}
$$

for all $x \in \Omega$ and all $\xi=\left(\xi_{1}, \ldots, \xi_{n}\right) \in \mathbb{R}^{n}$. 
We consider the quadratic form $Q$ :

$$
Q(u, v)=\int_{\Omega} \sum_{k, l=1}^{n} a_{k l}(x) \frac{\partial u}{\partial x_{k}}(x) \frac{\partial v}{\partial x_{l}}(x) d x
$$

with domain $H^{1}(\Omega) \hookrightarrow L_{2}(\Omega)$.

Lemma 4.1. The quadratic form (4.2) with domain of definition $H^{1}(\Omega)$ is a Dirichlet form.

Proof. Clearly $Q$ is non-negative and symmetric. According to M. FukUshima, Y. Oshima and M. TAKedA, see [29, Subsection 1.1], it remains to prove that $Q$ is closed and Markovian. Recall $Q$ is closed if $H^{1}(\Omega)$ equipped with the metric generated by the quadratic form $Q_{1}(u, v)=Q(u, v)+(u, v)_{L_{2}(\Omega)}$ is complete. This is clear in our case since we find constants $c_{1}, c_{2}>0$ such that

$$
c_{1}\left\|u\left|H^{1}(\Omega)\left\|^{2} \leq Q_{1}(u, u) \leq c_{2}\right\| u\right| H^{1}(\Omega)\right\|^{2}
$$

for all $u \in H^{1}(\Omega)$. The first inequality in (4.3) is an immediate consequence of the first inequality in (4.1), whereas the second inequality in (4.3) is a consequence of Hölder's inequality and of the boundedness of the functions $\left(a_{k l}\right)_{k, l=1, \ldots, n}$.

To see finally that $Q$ is Markovian, i. e. the unit contraction operates on $Q$, we follow the technique from [29, Examples 1.2.1 and 1.2.3]. We know that any function $u \in L_{2}(\Omega)$ such that $\frac{\partial u}{\partial x_{k}} \in L_{2}(\Omega)$ for all $k=1, \ldots, n$, has the property that there is a version $u_{0}$ of $u$, i.e. $u_{0}=u$ almost everywhere, such that $u_{0}$ is absolutely continuous on almost all straight lines which are parallel to the coordinate axes and the derivatives $\frac{\partial u_{0}}{\partial x_{k}}$ in the ordinary sense (which exist almost everywhere on $\Omega$ ) are in $L_{2}(\Omega)$, see V. G. MAZ'JA [43, Theorem 1.1.3/1, page 8] and cf. [29].

For each $\beta>0$ there exists a real function $\varphi_{\beta}: \mathbb{R} \rightarrow \mathbb{R}$ such that $\varphi_{\beta}(t)=t$ for all $t \in[0,1],-\beta \leq \varphi_{\beta}(t) \leq 1+\beta$ for all $t \in \mathbb{R}$ and

$$
0 \leq \varphi_{\beta}\left(t^{\prime}\right)-\varphi_{\beta}(t) \leq t^{\prime}-t \text { whenever } t<t^{\prime} .
$$

Such a function can easily be constructed, see [29, Problem 1.2.1].

For any $u \in H^{1}(\Omega)$ it follows $\varphi_{\beta}(u) \in H^{1}(\Omega)$ and

$$
\begin{aligned}
Q\left(\varphi_{\beta}(u), \varphi_{\beta}(u)\right) & =\sum_{k, l=1}^{n} \int_{\Omega} a_{k l}(x) \cdot\left|\varphi_{\beta}^{\prime}(u(x))\right|^{2} \frac{\partial u}{\partial x_{k}}(x) \frac{\partial u}{\partial x_{l}}(x) d x \\
& \leq Q(u, u)
\end{aligned}
$$

where the last inequality is a consequence of $0 \leq \varphi_{\beta}^{\prime}(t) \leq 1,\left|\varphi_{\beta}(t)-\varphi_{\beta}(s)\right| \leq|t-s|$ and $\left|\varphi_{\beta}(t)\right| \leq t$.

Since $Q$ is closed the inequality (4.4) is equivalent to the fact that $Q$ is Markovian, see $[29$, page $4-5]$. 


\subsection{Subordination in the sense of Bochner}

Subordination is a technique to obtain new semigroups from a given one. On the level of infinitesimal generators, subordination gives rise to a functional calculus. It was S. Bochner who developed these ideas in his 1949 paper [10] and in his monograph [11]. Standard references are the monographs of C. BerG, G. Forst [9], and of S. Bochner [11] and the papers of R. S. Phillips [45], F. Hirsch [33] and [34], C. Berg, Kh. Boyadzhiev and R. De Laubenfels [8], and R. L. Schilling [47] and [48], see also [35].

Let $\left(T_{t}\right)_{t \geq 0}$ be a strongly continuous semigroup on $L_{p}\left(\mathbb{R}^{n}\right)$ with generator $(A, D(A))$ and let $\left(\mu_{t}\right)_{t \geq 0}$ be a convolution semigroup of sub-probability measures supported on $[0, \infty)$. It is well known that these convolution semigroups are in a one-to-one correspondence with Bernstein functions. This correspondence is given by

$$
\int_{[0, \infty)} e^{-s x} \mu_{t}(d s)=e^{-t f(x)}, \quad t, x \geq 0 .
$$

In this situation the Bochner integral

$$
T_{t}^{f} u:=\int_{0}^{\infty} T_{s} u \mu_{t}(d s), \quad u \in L_{p}\left(\mathbb{R}^{n}\right),
$$

is well - defined and gives a strongly continuous semigroup on $L_{p}\left(\mathbb{R}^{n}\right)$. Properties like contractivity, Markov or Feller property are passed over from $\left(T_{t}\right)_{t \geq 0}$ to $\left(T_{t}^{f}\right)_{t \geq 0}$.

Let $\left(T_{t}\right)_{t \geq 0}$ be a strongly continuous semigroup on $L_{p}\left(\mathbb{R}^{n}\right)$ and let $\left(\mu_{t}\right)_{t \geq 0}$ be a vaguely continuous convolution semigroup of sub - probability measures on $[0, \infty)$. The semigroup $\left(T_{t}^{f}\right)_{t>0}$ given by (4.5) is called subordinate semigroup.

Its generator is denoted by $\left(A^{f}, D\left(A^{f}\right)\right)$.

For $f$ to be a Bernstein function it is necessary and sufficient to satisfy the following Lévy - Khinchin - type representation

$$
f(x)=a+b x+\int_{(0, \infty)}\left(1-e^{-t x}\right) \mu(d t)
$$

with $a, b \geq 0$ and a measure $\mu$ on $(0, \infty)$ such that $\int_{(0, \infty)} t /(1+t) \mu(d t)<\infty$.

The set of complete Bernstein functions consists of those Bernstein functions $f$ satisfying

$$
\mu(d t)=m(t) d t, \quad m(t)=\int_{(0, \infty)} e^{-r t} \rho(d r)
$$

with a measure $\rho$ on $(0, \infty)$ such that $\int_{(0, \infty)}(1+t)^{-1} \frac{\rho(d t)}{t}<\infty$.

It is not hard to see that any complete Bernstein function $f$ has the representation

$$
f(x)=a+b x+\int_{(0, \infty)} \frac{x}{t+x} \frac{\rho(d t)}{t}, \quad x \geq 0 .
$$

Examples for complete Bernstein functions are the fractional powers, $f_{\alpha}(x)=x^{\alpha}$ $(0 \leq \alpha \leq 1)$ or the logarithm $f(x)=\log (1+x)$. 
Using (4.6) and (4.7) one can obtain representation formulae for $A^{f}$. This problem was first investigated by R. S. PHILlips in [45] for general Bernstein functions. Here we follow F. Hirsch [33], [34], C. Berg, Kh. Boyadzhiev and R. DeLaubenfels [8], R. L. Schilling [48], where it was shown (independently) that for any complete Bernstein function $f$

$$
A^{f} u=-a u+b A u+\int_{(0, \infty)} A(\lambda \mathrm{id}-A)^{-1} u \frac{\rho(d \lambda)}{\lambda}, \quad u \in D(A),
$$

holds. This is a straightforward generalization of Balakrishnan's formula for fractional powers, see A. V. Balakrishnan [5] and K. Yosida [61, Chapter IX.11], in the sense that

$$
\left.A^{\left(x^{\alpha}\right)}\right|_{D(A)}=-\left.(-A)^{\alpha}\right|_{D(A)}, \quad 0<\alpha \leq 1 .
$$

Remark 4.2. Subordination has a stochastic interpretation. Suppose that we may associate with the semigroup $\left(T_{t}\right)_{t \geq 0}$ a Markov process $\left(X_{t}\right)_{t \geq 0}$ and denote by $\left(Y_{t}\right)_{t \geq 0}$ the Markov process associated with the convolution semigroup $\left(\mu_{t}\right)_{t \geq 0}$. The stochastic process associated with the semigroup $\left(T_{t}^{f}\right)_{t \geq 0}$ is given as a time-changed process, namely by $Z_{t}(\omega)=X_{Y_{t}(\omega)}(\omega)$.

Assume that $\left(T_{t}\right)_{t \geq 0}$ is a sub-Markovian semigroup with generator $(A, D(A))$ and corresponding Dirichlet form $(q, D(q))$. By subordination - as above $f$ denotes a Bernstein function - we get the subordinate semigroup $\left(T_{t}^{f}\right)_{t \geq 0}$ which is again subMarkovian. Thus by the general theory of Dirichlet forms there exists a corresponding Dirichlet from $q^{f}$ with domain $D\left(q^{f}\right)$ and generator $\left(A^{f}, D\left(A^{f}\right)\right)$. As usual $D\left(q^{f}\right)=$ $D\left(\left(-A^{f}\right)^{1 / 2}\right)$.

Remark 4.3. If $f$ is a complete Bernstein function then from Theorem 5.3 in [47] we have

$$
\left\|u\left|L_{2}(\Omega)\left\|^{2} \leq c q(u, u) \Longrightarrow\right\| u\right| L_{2}(\Omega)\right\|^{2} \leq \frac{c}{f(1)} q^{f}(u, u)
$$

for all $u \in D(q)$. The latter inequality holds also on $D\left(q^{f}\right)$ since we have the dense inclusions $D(A) \subset D(q)$ and $D(A) \subset D\left(q^{f}\right)$.

We will restrict ourselves to the case $f(x)=x^{\alpha}, 0<\alpha<1$, and write $\left(T_{t}^{(\alpha)}\right)_{t \geq 0}$, $q^{(\alpha)}$ and $A^{(\alpha)}$ instead of the clumsier $\left(T_{t}^{{ }^{*}}\right)_{t \geq 0}$ etc.

In fact we deal with fractional powers of the operator $-A$.

Using complex interpolation R. SeELEY determined in [50, Theorem 4.1] the domains of fractional powers of elliptic differential operators under regular boundary conditions.

Let $\Lambda$ be a positive operator acting in a Hilbert space. If there exist two positive numbers $\eta$ and $C$ such that $\Lambda^{i t}$ is a bounded operator for $-\eta \leq t \leq \eta$ and $\left\|\Lambda^{i t}\right\| \leq C$ then $\Lambda$ is said to have bounded imaginary powers, see for example [4, Section III.4.7]. 
If $\Lambda$ has bounded imaginary powers then for any two complex numbers $\alpha, \beta$ such that $0 \leq \operatorname{Re} \alpha<\operatorname{Re} \beta<\infty$, and any $0<\theta<1$ we have by complex interpolation

$$
\left[D\left(\Lambda^{\alpha}\right), D\left(\Lambda^{\beta}\right)\right]_{\theta}=D\left(\Lambda^{(1-\theta) \alpha+\theta \beta}\right),
$$

see [52, Theorem 1.15.3] for a proof and details.

All the above considerations in this subsection are of some general nature. We will return to a concrete Dirichlet form related to the form (4.2) in the next subsection.

\subsection{A Weyl decomposition for $H^{\alpha}(\Omega)$}

Let $Q$ be the same Dirichlet form on $H^{1}(\Omega)$ as in (4.2) where $\Omega$ and $\left(a_{k l}\right)_{k, l=1, \ldots, n}$ have the same meaning as in Subsection 4.1.

Let $\gamma>0$ be fixed. We consider the quadratic form $q$ defined by

$$
q(u, v)=Q(u, v)+\gamma(u, v)_{L_{2}(\Omega)}
$$

having domain $H^{1}(\Omega) \hookrightarrow L_{2}(\Omega)$. In fact

$$
q(u, v)=\int_{\Omega} \sum_{k, l=1}^{n} a_{k l}(x) \frac{\partial u}{\partial x_{k}}(x) \frac{\partial v}{\partial x_{l}}(x) d x+\gamma \int_{\Omega} u(x) v(x) d x .
$$

We obtain the following counterpart of [50, Theorem 4.1].

Theorem 4.4. Let $n-1 \leq d<n$ and let $\Omega$ be a bounded $(\varepsilon, \delta)$ domain in $\mathbb{R}^{n}$ such that $\Gamma=\partial \Omega$ is a d-set. Let $q$ be the Dirichlet form (4.10) defined on $H^{1}(\Omega)$ and let $q^{(\alpha)}$ be the Dirichlet form obtained by subordination with respect to the fractional power $f(x)=x^{\alpha}, 0<\alpha<1$. Then

$$
D\left(q^{(\alpha)}\right)=H^{\alpha}(\Omega) .
$$

Proof. Let $A$ be the generator of the Dirichlet form (4.10), i. e. the unique nonpositive self adjoint operator on $H^{1}(\Omega)$ such that $D(q)=D\left((-A)^{1 / 2}\right)$ and such that for all $u, v \in D(q)=H^{1}(\Omega)$

$$
q(u, v)=\left((-A)^{1 / 2} u,(-A)^{1 / 2} v\right)_{L_{2}(\Omega)} .
$$

Since

$$
(-A u, u)_{L_{2}(\Omega)} \geq \gamma\left\|u \mid L_{2}(\Omega)\right\|^{2}
$$

the operator $-A$ is positive, self adjoint and $-A \geq \gamma$. Thus we may use [4, Example III.4.7.3, page 164] to conclude that $-A$ has bounded imaginary powers.

Applying (4.9) we get by complex interpolation

$$
\left[L_{2}(\Omega), D\left((-A)^{1 / 2}\right)\right]_{\alpha}=D\left((-A)^{\alpha / 2}\right) .
$$

On the other hand we know $D\left((-A)^{1 / 2}\right)=D(q)=H^{1}(\Omega)$ and $D\left((-A)^{\alpha / 2}\right)=$ $D\left(q^{(\alpha)}\right)$. 
Moreover, $\left[L_{2}(\Omega), H^{1}(\Omega)\right]_{\alpha}=H^{\alpha}(\Omega)$ as a consequence of the corresponding result on $\mathbb{R}^{n}$, see [52], and of the fact that there is an extension operator for Sobolev spaces of fractional order on $(\varepsilon, \delta)$ domains.

The desired conclusion is now a simple application of (4.11).

Remark 4.5. It is obvious that $q_{D}:=q$ satisfies $q(u, u) \geq \gamma\left\|u \mid L_{2}(\Omega)\right\|^{2}$ on $\stackrel{\circ}{H^{1}}(\Omega)$. By (4.8) it follows

$$
\left\|u \mid L_{2}(\Omega)\right\|^{2} \leq c q^{(\alpha)}(u, u) \text { for all } u \in \stackrel{\circ}{H^{\alpha}}(\Omega)
$$

for some constant $c>0$. Thus $q^{(\alpha)}$ defines a scalar product that is equivalent to the canonical one $(\cdot, \cdot)_{\alpha}$ of $\stackrel{\circ}{H^{\alpha}}(\Omega)$.

Our aim is to show here how one can get a Weyl type decomposition of $H^{\alpha}(\Omega)$ with respect to the Dirichlet form $q^{(\alpha)}$.

The result we will obtain is the counterpart for $(\varepsilon, \delta)$ domains having boundary $\partial \Omega$ a $d$-set with $n-1 \leq d<n$ of a result obtained by the second named author and R. L. SCHILling in [36].

We put

$$
q_{\lambda}^{(\alpha)}(u, v)=\left((\lambda-A)^{\alpha / 2} u,(\lambda-A)^{\alpha / 2} v\right)_{L_{2}(\Omega)}, \quad \lambda \geq 0,
$$

where $A$ is the generator of $q$.

Clearly $\left(q_{\lambda}^{(\alpha)}, H^{\alpha}(\Omega)\right)$ is again a Dirichlet form and for $\lambda>0$ the form $q_{\lambda}^{(\alpha)}(\cdot, \cdot)$ is a scalar product, see (4.8), which is equivalent to the one of $H^{\alpha}(\Omega)$. Moreover, the quadratic forms $q_{\lambda}^{(\alpha)}(\cdot, \cdot)$ and $\left(A^{\alpha / 2}(\cdot), A^{\alpha / 2}(\cdot)\right)_{L_{2}(\Omega)}+\lambda(\cdot, \cdot)_{L_{2}(\Omega)}$ are equivalent. By $(4.12)$ on the space $\stackrel{\circ}{H}^{\alpha}(\Omega)$ this remains true even for $q_{0}^{(\alpha)}(\cdot, \cdot)$.

For $0<\alpha \leq 1$ and $\lambda \geq 0$ we call the functions in

$$
X_{\lambda}^{\alpha}(\Omega)=\left\{u \in H^{\alpha}(\Omega): q_{\lambda}^{(\alpha)}(u, v)=0 \text { for all } v \in \stackrel{\circ}{H}^{\alpha}(\Omega)\right\}
$$

$q_{\lambda}^{(\alpha)}$-harmonic functions. By the definition of $\stackrel{\circ}{H}^{\alpha}(\Omega)$ one has also

$$
X_{\lambda}^{\alpha}(\Omega)=\left\{u \in H^{\alpha}(\Omega): q_{\lambda}^{(\alpha)}(u, \varphi)=0 \text { for all } \varphi \in C_{0}^{\infty}(\Omega)\right\} .
$$

We are able now to state the main result of this section.

Theorem 4.6. Let $n-1 \leq d<n$ and let $\Omega$ be a bounded $(\varepsilon, \delta)$ domain in $\mathbb{R}^{n}$ such that $\Gamma=\partial \Omega$ is a $d$-set. For all $0<\alpha \leq 1$ and all $\lambda \geq 0$ one has the orthogonal decomposition

$$
H^{\alpha}(\Omega)=X_{\lambda}^{\alpha}(\Omega) \oplus_{q_{\lambda}^{(\alpha)}} \stackrel{\circ}{H}^{\alpha}(\Omega) .
$$

Moreover, if $\alpha>\frac{n-d}{2}$ the decomposition is non-trivial in the sense that $X_{\lambda}^{\alpha}(\Omega) \neq\{0\}$ and there is a canonical isomorphism

$$
\Pi_{\lambda}^{(\alpha)}: H^{\alpha-\frac{n-d}{2}}(\partial \Omega) \longrightarrow X_{\lambda}^{\alpha}(\Omega),
$$


where $H^{\sigma}(\partial \Omega)=B_{22}^{\sigma}(\partial \Omega)$.

Proof. Clearly $\stackrel{\circ}{H}^{\alpha}(\Omega)=\left\{v \in H^{\alpha}(\Omega): \operatorname{tr}_{\partial \Omega} v=0\right\}$, see Theorem 3.5, and $X_{\lambda}^{\alpha}(\Omega)$ are closed subspaces of $H^{\alpha}(\Omega)$. For all $u \in H^{\alpha}(\Omega)$ the condition $q_{\lambda}^{(\alpha)}(u, v)=0$ for all $v \in \stackrel{\circ}{H}^{\alpha}(\Omega)$ implies $u \equiv 0$ so that the decomposition is orthogonal.

By Theorem 3.9 one cannot expect non-trivial decompositions if $\alpha<\frac{n-d}{2}$.

As it was already mentioned, the case $\alpha=\frac{n-d}{2}$ will not be treated here. So let us assume that $\frac{n-d}{2}<\alpha \leq 1$. To show the existence of the isomorphism $\Pi_{\lambda}^{(\alpha)}$ as stated in the theorem one may follow the proof of [36, Theorem 4.1] inserting the results from the previous section.

By Theorem 3.3 there exists for any $\varphi \in H^{\alpha-\frac{n-d}{2}}(\partial \Omega)$ a function $f \in H^{\alpha}(\Omega)$ such that $\operatorname{tr}_{\partial \Omega} f=\varphi$. We define a linear functional

$$
L_{\lambda, f}^{\alpha}: H^{\alpha}(\Omega) \longrightarrow \mathbb{C} \quad \text { by } \quad L_{\lambda, f}^{\alpha}(v)=q_{\lambda}^{(\alpha)}(f, v) \quad \text { for } \quad v \in H^{\alpha}(\Omega) .
$$

By our assumptions $q_{\lambda}^{(\alpha)}(\cdot, \cdot)$ is for all $\lambda \geq 0$ a scalar product on $\stackrel{\circ}{H}^{\alpha}(\Omega)$ which is equivalent to the original one in $\stackrel{\circ}{H}^{\alpha}(\Omega)$. An application of the Lax-Milgram theorem shows that there exists a unique element $\omega_{\lambda, f} \in \stackrel{\circ}{H^{\alpha}}(\Omega)$ such that

$$
q_{\lambda}^{(\alpha)}\left(\omega_{\lambda, f}, v\right)=L_{\lambda, f}^{\alpha}(v) \quad \text { for all } v \in \stackrel{\circ}{H}^{\alpha}(\Omega) .
$$

We define $u_{\lambda, f}=\omega_{\lambda, f}-f$.

Step 1. The element $u_{\lambda, f}$ belongs to $X_{\lambda}^{\alpha}(\Omega)$. Indeed, for any $v \in \stackrel{\circ}{H^{\alpha}}(\Omega)$ we get:

$$
q_{\lambda}^{(\alpha)}\left(u_{\lambda, f}, v\right)=q_{\lambda}^{(\alpha)}\left(\omega_{\lambda, f}, v\right)-q_{\lambda}^{(\alpha)}(f, v)=L_{\lambda, f}^{\alpha}(v)-q_{\lambda}^{(\alpha)}(f, v)=0 .
$$

Step 2. $u_{\lambda, f}$ depends only on $\varphi=\operatorname{tr}_{\partial \Omega} f$ and the map $\varphi \mapsto u_{\lambda, \varphi}:=u_{\lambda, f}$ is linear.

Let $f_{1}, f_{2} \in H^{\alpha}(\Omega)$ such that $f_{1} \neq f_{2}$ but $\operatorname{tr}_{\partial \Omega} f_{1}=\operatorname{tr}_{\partial \Omega} f_{2}=\varphi$. Thus $\operatorname{tr}_{\partial \Omega}\left(f_{1}-f_{2}\right)=$ 0 which means, by Theorem 3.5, $f_{1}-f_{2} \in \stackrel{\circ}{H}^{\alpha}(\Omega)$.

Each $f_{j}, j=1,2$, has an orthogonal decomposition $f_{j}=u_{\lambda, f_{j}}+\omega_{\lambda, f_{j}}, j=1,2$, where $u_{\lambda, f_{j}} \in X_{\lambda}^{\alpha}(\Omega)$ and $\omega_{\lambda, f_{j}} \in \stackrel{\circ}{H}^{\alpha}(\Omega)$.

For every $v \in \stackrel{\circ}{H}^{\alpha}(\Omega)$ we have:

$$
\begin{aligned}
q_{\lambda}^{(\alpha)}\left(f_{1}-f_{2}, v\right) & =q_{\lambda}^{(\alpha)}\left(u_{\lambda, f_{1}}-u_{\lambda, f_{2}}, v\right)+q_{\lambda}^{(\alpha)}\left(\omega_{\lambda, f_{1}}-\omega_{\lambda, f_{2}}, v\right) \\
& =0+q_{\lambda}^{(\alpha)}\left(\omega_{\lambda, f_{1}}-\omega_{\lambda, f_{2}}, v\right) .
\end{aligned}
$$

Consequently, $\left(f_{1}-f_{2}\right)-\left(\omega_{\lambda, f_{1}}-\omega_{\lambda, f_{2}}\right) \in X_{\lambda}^{\alpha}(\Omega)$.

But $f_{1}-f_{2} \in \stackrel{\circ}{H}^{\alpha}(\Omega)$ and $\omega_{\lambda, f_{1}}-\omega_{\lambda, f_{2}} \in \stackrel{\circ}{H}^{\alpha}(\Omega)$ and so $f_{1}-f_{2}=\omega_{\lambda, f_{1}}-\omega_{\lambda, f_{2}}$ or $u_{\lambda, f_{1}}=u_{\lambda, f_{2}}$.

The linearity $\varphi \mapsto u_{\lambda, \varphi}$ is clear. So far we have proved that

$$
\Pi_{\lambda}^{(\alpha)}: H^{\alpha-\frac{n-d}{2}}(\partial \Omega) \longrightarrow X_{\lambda}^{\alpha}(\Omega), \quad \varphi \longmapsto u_{\lambda, \varphi}
$$


is a well defined, linear operator.

Step 3. The mapping $\Pi_{\lambda}^{(\alpha)}$ is bijective.

Step 3.1. Injectivity. Assume $\Pi_{\lambda}^{(\alpha)} \varphi=0$ for some $\varphi \in H^{\alpha-\frac{n-d}{2}}(\partial \Omega)$. Let $f \in$ $H^{\alpha}(\Omega)$ such that $\operatorname{tr}_{\partial \Omega} f=\varphi$. But $\Pi_{\lambda}^{(\alpha)} \varphi=u_{\lambda, \varphi}=\omega_{\lambda, f}-f$. It follows $\omega_{\lambda, f}=f \in$ $\stackrel{\circ}{H}^{\alpha}(\Omega) \cap H^{\alpha}(\Omega)$ which is by Theorem $3.5 \operatorname{tr}_{\partial \Omega} f=0$ or $\varphi=0$.

Step 3.2. Surjectivity. Let $u \in X_{\lambda}^{\alpha}(\Omega) \subset H^{\alpha}(\Omega)$. By the trace theorem, Theorem 3.3, there exists $\varphi=\operatorname{tr}_{\partial \Omega} u \in H^{\alpha-\frac{n-d}{2}}(\partial \Omega)$. We define $u_{\lambda, \varphi}=\Pi_{\lambda}^{(\alpha)} \varphi$. Clearly $u_{\lambda, \varphi}-u \in X_{\lambda}^{\alpha}(\Omega)$.

On the other hand $u_{\lambda, \varphi}-u$ has the property $\operatorname{tr}_{\partial \Omega}\left(u_{\lambda, \varphi}-u\right)=0$ and this is, again by Theorem $3.5, u_{\lambda, \varphi}-u \in \stackrel{\circ}{H}^{\alpha}(\Omega)$.

Consequently $u_{\lambda, \varphi}-u \in X_{\lambda}^{\alpha}(\Omega) \cap \stackrel{\circ}{H^{\alpha}}(\Omega)$ and this is $u_{\lambda, \varphi}=u$, i. e. the surjectivity.

Step 4. The mapping $\Pi_{\lambda}^{(\alpha)}: H^{\alpha-\frac{n-d}{2}}(\partial \Omega) \rightarrow X_{\lambda}^{\alpha}(\Omega)$ is continuous (the Hilbert spaces are equipped with their canonical scalar products). Since $H^{\alpha}(\Omega)$ is the orthogonal sum of two closed subspaces the projections:

$$
\pi_{1}: H^{\alpha}(\Omega) \longrightarrow \stackrel{\circ}{H}^{\alpha}(\Omega) \text { and } \pi_{2}: H^{\alpha}(\Omega) \longrightarrow X_{\lambda}^{\alpha}(\Omega)
$$

are orthogonal projections, hence continuous. Let $\widetilde{\gamma}$ be the linear right inverse of $\operatorname{tr}_{\partial \Omega}: H^{\alpha}(\Omega) \rightarrow H^{\alpha-\frac{n-d}{2}}(\partial \Omega)$, see Theorem 3.3. We know that $\widetilde{\gamma}$ is continuous and this implies the continuity of $\Pi_{\lambda}^{(\alpha)}=\pi_{2} \circ \widetilde{\gamma}$.

The proof is now complete.

\subsection{An analogue of the Douglas integral}

Let again $n-1 \leq d<n$, let $\Omega \subset \mathbb{R}^{n}$ be a bounded $(\varepsilon, \delta)$ domain having boundary $\Gamma=\partial \Omega$ which is a $d$-set and let $\mu=\mathcal{H}^{d} \mid \Gamma$.

Let $\alpha \in \mathbb{R}$ such that $\frac{n-d}{2}<\alpha<1+\frac{n-d}{2}$. According to Definition 2.5 the norm in $H^{\alpha-\frac{n-d}{2}}(\partial \Omega)$ is given by

$$
\left\|u\left|H^{\alpha-\frac{n-d}{2}}(\partial \Omega)\|=\| u\right| L_{2}(\partial \Omega)\right\|+\left(\iint_{|x-y|<1} \frac{|u(x)-u(y)|^{2}}{|x-y|^{2 d+2 \alpha-n}} d \mu(x) d \mu(y)\right)^{1 / 2}
$$

For $\frac{n-d}{2}<\alpha \leq 1$ let us denote

$$
\begin{aligned}
S^{(\alpha)}(\varphi, \psi)= & \int_{\partial \Omega} \varphi(x) \psi(x) d \mu(x) \\
& +\iint_{|x-y|<1} \frac{(\varphi(x)-\varphi(y)) \cdot(\psi(x)-\psi(y))}{|x-y|^{2 d+2 \alpha-n}} d \mu(x) d \mu(y)
\end{aligned}
$$

for any $\varphi, \psi \in H^{\alpha-\frac{n-d}{2}}(\partial \Omega)$.

It is clear that the quadratic form $S^{(\alpha)}$ having domain of definition $D\left(S^{(\alpha)}\right)=$ $H^{\alpha-\frac{n-d}{2}}(\partial \Omega)$ is a non-negative, symmetric and closed form on $L_{2}(\partial \Omega)$. Moreover, 
using the same technique as in the proof of Lemma 4.1 the quadratic form $S^{(\alpha)}$ is also Markovian and hence a Dirichlet form on $L_{2}(\partial \Omega)$.

In particular the unit contraction operator $N_{\partial \Omega} \varphi=(0 \vee \varphi) \wedge 1$ leaves the form domain $D\left(S^{(\alpha)}\right)$ invariant and operates continuously thereon,

$$
S^{(\alpha)}\left(N_{\partial \Omega} \varphi, N_{\partial \Omega} \varphi\right) \leq S^{(\alpha)}(\varphi, \varphi), \quad \varphi \in D\left(S^{(\alpha)}\right)=H^{\alpha-\frac{n-d}{2}}(\partial \Omega) .
$$

Denote by $N_{\Omega}, N_{\Omega} u=(0 \vee u) \wedge 1$ the unit contraction defined for functions $u: \bar{\Omega} \rightarrow \mathbb{R}$.

Let $C(\bar{\Omega})$ the space of all restrictions to $\bar{\Omega}$ of functions from $C\left(\mathbb{R}^{n}\right)$ and let $\widetilde{H}^{\alpha}(\Omega)$ be the closure of $C(\bar{\Omega}) \cap H^{\alpha}(\Omega)$ in the norm of $H^{\alpha}(\Omega)$.

Theorem 4.7. Let $n-1 \leq d<n$, let $\Omega \subset \mathbb{R}^{n}$ be a bounded $(\varepsilon, \delta)$ domain having boundary $\Gamma=\partial \Omega$ which is a $d-$ set and let $\frac{n-d}{2}<\alpha \leq 1$. Then $\widetilde{H}^{\alpha}(\Omega)=H^{\alpha}(\Omega)$.

Proof. Let $\varepsilon>0$ and $u \in H^{\alpha}(\Omega)$. Let $E u \in H^{\alpha}(\Omega)$ according to Theorem 2.3. Then there exists an $\psi_{\varepsilon} \in \mathcal{S}\left(\mathbb{R}^{n}\right)$ such that $\left\|E u-\psi_{\varepsilon} \mid H^{\alpha}(\Omega)\right\|<\varepsilon$.

Taking into account the fact that the norm in $H^{\alpha}(\Omega)$ is initially defined by restriction, see (2.4), we have $\left\|u-\left.\psi_{\varepsilon}\right|_{\Omega} \mid H^{\alpha}(\Omega)\right\|<\varepsilon$ and this completes the proof.

Lemma 4.8. Let $\operatorname{tr}_{\partial \Omega}$ be the trace operator on $H^{\alpha}(\Omega)$ and let $N_{\partial \Omega}, N_{\Omega}$ be the unit contractions on $\partial \Omega$ and $\Omega$. Then

$$
\operatorname{tr}_{\partial \Omega}\left(N_{\Omega} u\right)=N_{\Omega}\left(\operatorname{tr}_{\partial \Omega} u\right) \quad \text { for any } u \in H^{\alpha}(\Omega) .
$$

Proof. For $u \in C(\bar{\Omega}) \cap H^{\alpha}(\Omega)$ the assertion is straightforward according to the definition of the trace, see (3.1) and Definition 3.1. The conclusion follows now by standard density arguments.

We know from Theorem 3.3 that $\operatorname{tr}_{\partial \Omega}: H^{\alpha}(\Omega) \rightarrow H^{\alpha-\frac{n-d}{2}}(\partial \Omega)$ is a bounded linear surjection with a right continuous inverse.

Let $q^{(\alpha)}, \Pi_{\lambda}^{(\alpha)}$ and $X_{\lambda}^{\alpha}(\Omega)$ having the same meaning as in Theorem 4.6.

Then

$$
\mathcal{C}_{\lambda}^{(\alpha)}(\varphi, \psi)=q_{\lambda}^{(\alpha)}\left(\Pi_{\lambda}^{(\alpha)} \varphi, \Pi_{\lambda}^{(\alpha)} \psi\right) \quad \text { if } \quad \varphi, \psi \in H^{\alpha-\frac{n-d}{2}}(\partial \Omega),
$$

defines on $H^{\alpha-\frac{n-d}{2}}(\partial \Omega)$ a bilinear form.

We know that $\Pi_{\lambda}^{(\alpha)}: H^{\alpha-\frac{n-d}{2}}(\partial \Omega) \rightarrow X_{\lambda}^{\alpha}(\Omega)$ is linear, continuous and bijective.

Since $\left(X_{\lambda}^{\alpha}(\Omega),(\cdot, \cdot)_{\alpha}\right)$ is a closed subspace of $H^{\alpha}(\Omega)$ it is itself a Hilbert space and there exist constants $c_{1}, c_{2}>0$ such that

$$
c_{1}\left\|\varphi\left|H^{\alpha-\frac{n-d}{2}}(\partial \Omega)\|\leq\| \Pi_{\lambda}^{(\alpha)} \varphi\right| H^{\alpha}(\Omega)\right\| \leq c_{2}\left\|\varphi \mid H^{\alpha-\frac{n-d}{2}}(\partial \Omega)\right\|
$$

and hence $\mathcal{C}_{\lambda}^{(\alpha)}$ is a closed form on $H^{\alpha-\frac{n-d}{2}}(\partial \Omega)$.

Moreover, we have the following theorem.

Theorem 4.9. The bilinear form $\mathcal{C}_{\lambda}^{(\alpha)}$ is a Dirichlet form on $H^{\alpha-\frac{n-d}{2}}(\partial \Omega)$. 
Proof. $\mathcal{C}_{\lambda}^{(\alpha)}$ being a closed form, it remains to prove the contraction property for the unit contraction $N_{\partial \Omega}$,

$$
\mathcal{C}_{\lambda}^{(\alpha)}\left(N_{\partial \Omega} \varphi, N_{\partial \Omega} \varphi\right) \leq \mathcal{C}_{\lambda}^{(\alpha)}(\varphi, \varphi) \text { for all } \varphi \in H^{\alpha-\frac{n-d}{2}}(\partial \Omega)
$$

In order to see this, let us first prove that

$$
N_{\Omega}\left(\Pi_{\lambda}^{(\alpha)} \varphi\right)=\Pi_{\lambda}^{(\alpha)}\left(N_{\partial \Omega} \varphi\right)+g_{\varphi}
$$

where $\Pi_{\lambda}^{(\alpha)}\left(N_{\partial \Omega} \varphi\right) \in X_{\lambda}^{\alpha}(\Omega), g_{\varphi} \in \stackrel{\circ}{H}^{\alpha}(\Omega)$. Since the decomposition on the right-hand side in (4.15) is unique it is sufficient to prove that the traces satisfy

$$
\operatorname{tr}_{\partial \Omega}\left(N_{\Omega}\left(\Pi_{\lambda}^{(\alpha)} \varphi\right)\right)=\operatorname{tr}_{\partial \Omega}\left(\Pi_{\lambda}^{(\alpha)}\left(N_{\partial \Omega} \varphi\right)\right) .
$$

Since $\operatorname{tr}_{\partial \Omega} \circ \Pi_{\lambda}^{(\alpha)}=$ id on $H^{\alpha-\frac{n-d}{2}}(\partial \Omega)$, see also Theorems 3.3 and 3.5, the equality (4.16) is in fact the assertion of Lemma 4.8.

Using (4.16) we find

$$
\begin{aligned}
\mathcal{C}_{\lambda}^{(\alpha)}(\varphi, \varphi)= & q_{\lambda}^{(\alpha)}\left(\Pi_{\lambda}^{(\alpha)} \varphi, \Pi_{\lambda}^{(\alpha)} \varphi\right) \\
\geq & q_{\lambda}^{(\alpha)}\left(N_{\Omega}\left(\Pi_{\lambda}^{(\alpha)} \varphi\right), N_{\Omega}\left(\Pi_{\lambda}^{(\alpha)} \varphi\right)\right) \\
= & q_{\lambda}^{(\alpha)}\left(\Pi_{\lambda}^{(\alpha)}\left(N_{\partial \Omega} \varphi\right), \Pi_{\lambda}^{(\alpha)}\left(N_{\partial \Omega} \varphi\right)\right) \\
& +2 q_{\lambda}^{(\alpha)}\left(\Pi_{\lambda}^{(\alpha)}\left(N_{\partial \Omega} \varphi\right), g_{\varphi}\right)+q_{\lambda}^{(\alpha)}\left(g_{\varphi}, g_{\varphi}\right) \\
\geq & \mathcal{C}_{\lambda}^{(\alpha)}\left(N_{\partial \Omega} \varphi, N_{\partial \Omega} \varphi\right)
\end{aligned}
$$

and this shows that $\mathcal{C}_{\lambda}^{(\alpha)}$ is a Dirichlet form on $H^{\alpha-\frac{n-d}{2}}(\partial \Omega)=\operatorname{tr}_{\partial \Omega} H^{\alpha}(\Omega)$.

We will need an embedding of Sobolev type for Besov spaces $B_{p p}^{\sigma}(\Gamma)$ on a $d$-set $\Gamma$, recall Definition 2.5.

Lemma 4.10. If $\Gamma$ is a $d$-set with $0<d<n$ then there exists an embedding

$$
B_{p p}^{s-\frac{n-d}{p}}(\Gamma) \longrightarrow L_{r}(\Gamma) \text { where } r=\frac{d p}{n-s p},
$$

where $1<p<\infty, \frac{n-d}{p}<s<1+\frac{n-d}{p}$ and $n-s p>0$.

Proof. The above embedding is a simple consequence of the embeddings

$$
E_{\Gamma}: B_{p p}^{s-\frac{n-d}{p}}(\Gamma) \longrightarrow H_{p}^{s}\left(\mathbb{R}^{n}\right) \text { and } \operatorname{Tr}^{\Gamma}: H_{p}^{s}\left(\mathbb{R}^{n}\right) \longrightarrow L_{r}(\Gamma) .
$$

The embedding $E_{\Gamma}$ is stated in Theorem 2.7, whereas the embedding $\operatorname{Tr}^{\Gamma}$ is due to D. R. Adams, see [2] and see also [40, Lemma 1, page 214].

Let us return to the Dirichlet form $\left(S^{(\alpha)}, H^{\alpha-\frac{n-d}{2}}(\partial \Omega)\right)$. The form $S^{(\alpha)}$ is in particular closed on $H^{\alpha-\frac{n-d}{2}}(\partial \Omega)$ so that $S_{\beta}^{(\alpha)}(\cdot, \cdot)=S^{(\alpha)}(\cdot, \cdot)+\beta(\cdot, \cdot)_{L_{2}(\partial \Omega)}$ is for any $\beta>0$ a scalar product which is equivalent to the scalar product in $H^{\alpha-\frac{n-d}{2}}(\partial \Omega)$. 
Similarly $\mathcal{C}_{\lambda, \beta}^{(\alpha)}(\cdot, \cdot)=\mathcal{C}_{\lambda}^{(\alpha)}(\cdot, \cdot)+\beta(\cdot, \cdot)_{L_{2}(\partial \Omega)}$ is also a scalar product which is equivalent to the scalar product in $H^{\alpha-\frac{n-d}{2}}(\partial \Omega)$, thus $S_{\beta}^{(\alpha)}$ and $\mathcal{C}_{\lambda, \beta}^{(\alpha)}$ are equivalent to each other.

By the Sobolev embedding from Lemma 4.10, applied to the space $H^{\alpha-\frac{n-d}{2}}(\partial \Omega)$, we get

$$
\left\|u\left|L_{r}(\partial \Omega)\|\leq c\| u\right| H^{\alpha-\frac{n-d}{2}}(\partial \Omega)\right) \| \quad \text { for any } \quad u \in H^{\alpha-\frac{n-d}{2}}(\partial \Omega),
$$

where $r=\frac{2 d}{n-2 \alpha}$. Note that $r>2$ since $\alpha>\frac{n-d}{2}$.

Consequently there exist constants $c, c^{\prime}>0$ such that

$$
\left\|u \mid L_{r}(\partial \Omega)\right\| \leq c S_{\beta}^{(\alpha)}(u, u) \text { for any } u \in H^{\alpha-\frac{n-d}{2}}(\partial \Omega)
$$

and

$$
\left\|u \mid L_{r}(\partial \Omega)\right\| \leq c^{\prime} \mathcal{C}_{\lambda, \beta}^{(\alpha)}(u, u) \text { for any } u \in H^{\alpha-\frac{n-d}{2}}(\partial \Omega) .
$$

Let $\left(T_{t}^{S_{\beta}^{(\alpha)}}\right)_{t \geq 0}$ and $\left(T_{t}^{\mathcal{C}_{\lambda, \beta}^{(\alpha)}}\right)_{t \geq 0}$ be the semigroups associated to the forms $S_{\beta}^{(\alpha)}$ and $\mathcal{C}_{\lambda, \beta}^{(\alpha)}$. Using [59, page 4] we get that both semigroups satisfy the estimates

$$
\left\|T_{t}^{S_{\beta}^{(\alpha)}}\right\|_{L_{1} \rightarrow L_{\infty}} \leq c_{\beta} \frac{e^{\beta t}}{t^{d /(d+2 \alpha-n)}} \quad \text { and }\left\|T_{t}^{\mathcal{C}_{\lambda, \beta}^{(\alpha)}}\right\|_{L_{1} \rightarrow L_{\infty}} \leq c_{\beta}^{\prime} \frac{e^{\beta t}}{t^{d /(d+2 \alpha-n)}}
$$

\section{Acknowledgements}

This work was supported through the DFG-project Ja522/7-1 Funktionenräume in der Theorie der stochastischen Prozesse.

It is a pleasure to give our thanks to Professor HANS TRIEBEL; this research was initiated after a discussion we have had with him in Jena in Summer 1998. We also thank Professor MAsatoshi FuKushima for valueable comments on reflecting diffusions.

\section{References}

[1] Adams, D. R., and Hedberg, L. I.: Function Spaces and Potential Theory, Springer-Verlag, Berlin, 1996

[2] Adams, D. R.: Traces of Potentials Arising from Translation Invariant Operators, Ann. Sc. Norm. Sup. Pisa 25 (1971), 203-217

[3] Adams, R. A.: Sobolev Spaces, Academic Press, 1975

[4] Amann, H.: Linear and Quasi-Linear Parabolic Problems, Vol. I Abstract Linear Theory, Monographs in Mathematics Vol. 89, Birkhäuser, Basel, 1995

[5] Balakrishnan, A. V.: Fractional Powers of Closed Operators and Semigroups Generated by Them, Pacific J. Math. 10 (1960), 419-437

[6] Bass, R.F., and Hsu, P.E.: The Semimartingale Structure of Reflecting Brownian Motion, Proc. Amer. Math. Soc. 108 (1990), 1007-1010

[7] Bass, R. F., and Hsu, P. E.: Some Potential Theory for Reflecting Brownian Motion in Hölder and Lipschitz Domains, Ann. Prob. 19 (1991), 486-508 
[8] Berg, C., Boyadzhiev, Kh., and delaubenfels, R.: Generation of Generators of Holomorphic Semigroups, J. Austral. Math. Soc. (Ser. A) 55 (1993), 246-269

[9] Berg, C., and Forst, G.: Potential Theory on Locally Compact Abelian Groups, Ergebnisse der Mathematik und ihre Grenzgebiete, II. Ser., Vol. 87, Springer-Verlag, Berlin, 1975

[10] Bochner, S.: Diffusion Equation and Stochastic Processes, Proc. Natl. Acad. Sci. 35 (1949), $368-370$

[11] Bochner, S.: Harmonic Analysis and the Theory of Probability, California Monographs in Math. Sci., University of California Press, Berkeley CA, 1955

[12] Caetano, A.: Approximation by Functions of Compact Support in Besov-Triebel-Lizorkin Spaces on Irregular Domains, Studia Math. 142 (2000), 47-63

[13] Cacciopoli, R.: Misure e Integrazione sugli Insiemi Dimesionalmente Orientati, Rend. Accad. Naz. dei Lincei 12 (1953), 3-11, 137-146

[14] Chen, Z. Q., Fitzsimmons, P.J., and Williams, R. J.: Reflecting Brownian Motions: Quasimartingales and Strong Cacciopoli Sets, Potential Analysis 2 (1993), 219-243

[15] De Giongi, E.: Su una Teoria Generale della Misure $(r-1)$ Dimensionale in uno Spazio ad $r$ Dimensioni, Ann. Mat. Pura Appl. IV Ser. 36 (1954), 191-213

[16] De Giorgi, E.: Nuovi Teoremi Relative alle Misure $(r-1)$ Dimensionale in Spazio ad $r$ Dimensioni, Ric. Mat. 4 (1955), 95-113

[17] De Vore, R. A., and Sharpley, R. C.: Besov Spaces on Domains in $\mathbb{R}^{d}$, Trans. Amer. Math. Soc. 335 (1993), 843-864

[18] Dunford, N., and Schwartz, J. T.: Linear Operators I, Pure Appl. Math. Vol. 7, Interscience Publishers, New York, 1957

[19] Edmunds, D. E., and Triebel, H.: Function Spaces, Entropy Numbers, Differential Operators, Cambridge University Press, 1996

[20] Evans, L. C., and Gariepy, R.F.: Measure Theory and Fine Properties of Functions, CRC Press, 1992

[21] Fajn, B. L.: Extensions from Anisotropic Sobolev Spaces, Trudy Math. Inst. Steklov 158 (1984), $248-273$ (in Russian)

[22] Falconer, K. J.: The Geometry of Fractal Sets, Cambridge University Press, 1985

[23] Falconer, K. J.: Fractal Geometry, Wiley, Chichester, 1990

[24] Falconer, K. J.: Techniques in Fractal Geometry, Wiley, Chicester, 1997

[25] Frazier, M., and Jawerth, B.: Decomposition of Besov Spaces, Indiana Univ. Math. J. 34 (1985), $777-799$

[26] Frazier, M., and Jawerth, B.: A Discrete Transform and Decomposition of Distribution Spaces, J. Funct. Anal. 93 (1990), 34-170

[27] Fukushima, M.: Dirichlet Forms, Cacciopoli Sets and the Skorohod Representation. In: Csiszar, Michaletzky (eds), Stochastic Differential and Difference Equations, pp. 59-66, Birkhäuser Verlag, Basel, 1997

[28] Fukushima, M.: Decompositions of Symmetric Diffusions and Related Topics in Analysis, Sugaku 50 (1998), 56-67 (in Japanese)

[29] Fukushima, M., Oshima, Y., and Takeda, M.: Dirichlet Forms and Symmetric Markov Processes, Walter de Gruyter Verlag, 1994

[30] Fukushima, M., and Tomisaki, M.: Reflecting Diffusions on Lipschitz Domains with Cusps Analytic Construction and Skorohod Representation, Potential Analysis 4 (1995), 377-408

[31] Fukushima, M., and Tomisaki, M.: Construction and Decomposition of Reflecting Diffusions on Lipschitz Domains with Hölder Cusps, Probab. Theory Relat. Fields 106 (1996), 521-557

[32] Giusti, E.: Minimal Surfaces and Functions of Bounded Variation, Monographs in Mathematics Vol. 80, Birkhäuser Verlag, 1984 
[33] Hirsch, F.: Domaines d'Opérateurs Représentés comme Intégrales de Resolvantes, J. Funct. Anal. 23 (1976), 239-264

[34] Hirsch, F.: Extension des Propriétés des Puissances Fractionaires. In: Sém. Theorie du Potentiel, Lecture Notes in Mathematics Vol. 563, pp. 100-120, Springer-Verlag, Berlin 1976

[35] JACOB, N.: Pseudo-Differential Operators and Markov Processes, I. Fourier Analysis and Semigroups (book manuscript)

[36] Jacob, N., and Schilling, R. L.: Some Dirichlet Spaces Obtained by Subordinate Reflected Diffusions, Rev. Math. Iberoamericana 15 (1999), 59-91

[37] Jerison, D., and Kenig, C.: Boundary Behavior of Harmonic Functions in Nontangentially Accessible Domains, Adv. Math. 46 (1982), 80-146

[38] Jones, P.W.: Quasiconformal Mappings and Extendability of Functions in Sobolev Spaces, Acta Math. 147 (1981), $71-88$

[39] Jonsson, A.: Besov Spaces on Closed Subsets of $\mathbb{R}^{n}$, Trans. Amer. Math. Soc. 341 (1994), $355-370$

[40] Jonsson, A., and Wallin, H.: Function Spaces on Subsets of $\mathbb{R}^{n}$, Math. Reports Vol. 2, Part 1, Harwood Acad. Publ., London, 1984

[41] Jonsson, A., and Wallin, H.: Boundary Value Problems and Brownian Motion on Fractals, Chaos, Solitons and Fractals 8 (1997), 191-205

[42] Marschall, J.: The Trace of Sobolev-Slobodeckij Spaces on Lipschitz Domains, Manuscripta Math. 58 (1987), $47-65$

[43] MaZ'JA, V. G.: Sobolev Spaces, Springer-Verlag, 1985

[44] Maz'JA, V. G., and Poborchi, S. B.: Differentiable Functions on Bad Domains, World Scientific, 1997

[45] Phillips, R. S.: On the Generation of Semigroups of Linear Operators, Pacific J. Math. 2 (1952), $343-369$

[46] Runst, T., and Sickel, W.: Sobolev Spaces of Fractional Order, Nemytskij Operators and Nonlinear Partial Differential Equations, Walter de Gruyter Verlag, Berlin, 1996

[47] Schilling, R. L.: On the Domain of the Generator of a Subordinate Semigroup. In: L. Kral et al (eds), Potential Theory - ICTP 94, Proceedings Int. Conf. Potential Theory, Kouty (CR), 1994, pp. 446-462, Walter de Gruyter Verlag, Berlin, 1996

[48] Schilling, R. L.: Subordination in the Sense of Bochner and a Related Functional Calculus, J. Austral. Math. Soc. (Ser. A) 64 (1998), 368-396

[49] Seeger, A.: A Note on Triebel-Lizorkin Spaces. In: Approximations and Function Spaces, Banach Centre Publ. 22 (1989), 391-400, PWN Polish Sci. Publ., Warszaw

[50] Seeley, R.: Interpolation in $L^{p}$ with Boundary Conditions, Studia Math. 44 (1972), 47-60

[51] Stein, E. M.: Singular Integrals and Differentiability Properties of Functions, Princeton University Press, Princeton NJ, 1970

[52] Triebel, H.: Interpolation Theory, Function Spaces, Differential Operators, North Holland, Amsterdam, 1978

[53] Triebel, H.: Theory of Function Spaces, Geest \& Portig, Leipzig, 1983

[54] Triebel, H.: Theory of Function Spaces II, Birkhäuser, Basel, 1992

[55] Triebel, H.: Fractals and Spectra, Birkhäuser, Basel, 1997

[56] Triebel, H.: Decompositions of Function Spaces, Progr. Nonlinear Differential Equations Appl. 35 (1999), $691-730$

[57] Triebel, H.: The Structure of Functions, Birkhäuser, Basel, 2001 (in press)

[58] Triebel, H., and Winkelvoss, H.: A Fourier Analytical Characterization of the Hausdorff Dimension of a Closed Set and of Related Lebesgue Spaces, Studia Math. 121 (1996), 149-166 
[59] Varopoulos, N.Th., Saloff-Coste, L., and Coulhon, T.: Analysis and Geometry on Groups, Cambridge University Press, 1992

[60] Wallin, H.: The Trace to the Boundary of Sobolev Spaces on a Snowflake, Manuscripta Math. 73 (1991), $117-125$

[61] Yosida, K.: Functional Analysis, Grundlehren der mathematischen Wissenschaften Vol. 123, Springer-Verlag, Berlin, 1980 ( $6^{\text {th }}$ edition)

[62] Ziemer, W. P.: Weakly Differentiable Functions, Springer-Verlag, 1989

Universität München

Mathematisches Institut

Theresienstrasse 39

D-80333 München

Germany

E-mail:

farkas@rz.mathematik.uni-muenchen.de
University of Wales at Swansea

Department of Mathematics

Singleton Park

Swansea SA2 8PP

United Kingdom

E-mail:

N.Jacob@swansea.ac.uk 